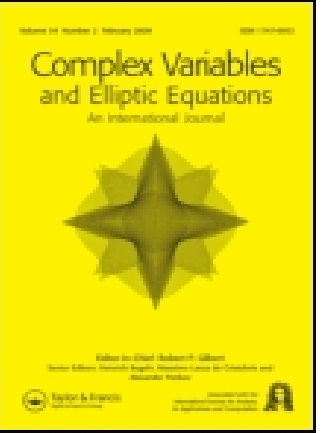

Complex Variables, Theory and Application: An International Journal

ISSN: 0278-1077 (Print) 1563-5066 (Online) Journal homepage: https://www.tandfonline.com/loi/gcov19

\title{
Anatolii asirovich gol'dberg
}

\section{A. Eremenkoa , I. Ostrovskii \& M. Sodin}

To cite this article: A. Eremenkoa, I. Ostrovskii \& M. Sodin (1998) Anatolii asirovich gol'dberg, Complex Variables, Theory and Application: An International Journal, 37:1-4, 1-51, DOI: $10.1080 / 17476939808815121$

To link to this article: https://doi.org/10.1080/17476939808815121

Published online: 29 May 2007.

Submit your article to this journal 주

Џlll Article views: 227

4 Citing articles: 4 View citing articles 5 


\title{
Anatolii Asirovich Gol'dberg
}

\author{
A. EREMENKO ${ }^{a, *}$, I. OSTROVSKIIb,d,t and M. SODIN ${ }^{c, d, \neq}$
}

a Department of Mathematics, Purdue University, West Lafayette, IN 47907, USA; 'D Department of Mathematics, Bilkent University, 06533 Bilkent, Ankara, Turkey; 'School of Mathematical Sciences, Tel Aviv University, Tel-Aviv 69978, Israel; 'Mathematical Division, B. Verkin Institute for Low Temperature Physics, Lenin's pr. 47, Kharkov 310164, Ukraine

(Received June 1997)

\section{INTRODUCTION}

Anatolii Asirovich Gol'dberg was born on April 2, 1930, in Kiev USSR. His father was a physician, his mother was a high school teacher. In 1933-41 they lived in Zaporozh'e, a city by Dnieper river. With the German Army nearing Zaporozh'e in 1941, his mother escaped with him to the East of the Soviet Union. After the war his family moved to the beautiful city of Lvov (=Lemberg, Lviv), the center of Galicia (=Galychina).

Gol'dberg graduated from the secondary school in 1947. By that time he resolved to become a mathematician having been strongly encouraged by Prof. Alexander S. Kovan'ko. Kovan'ko was the Chairman of the

\footnotetext{
- Suppurted by NSF grant DMS-9500636

† The second-named author thanks Professor Matts Essen and the Department of Mathematics of Uppsala University for their kind hospitality during his work on this paper

¥ The work of the third-named author was done in the framework of the INTAS research network 94-1474 Potential Theory, Complex Analysis and Their Applications to Ordinary and Partial Differential Equations
} 
Organizing Committee of the Lvov youth mathematical competition of 1947, the so-called Mathematical Olympiad, in which Gol'dberg won the first place. That year he entered the Department of Physics and Mathematics of Lvov University. In the 20-ies and 30-ies, while Lvov was in Poland, a famous mathematical school was formed in Lvov University around Hugo Steinhaus and Stefan Banach. During the Second World War this school appeared to be completely dispersed. The chain of mathematical tradition was broken and after the war mathematicians from various places of the Soviet Union came to work at the Lvov University. Among them were Boris V. Gnedenko, Alexander S. Kovan'ko, Yaroslav B. Lopatinskii, Ivan G. Sokolov, Lev I. Volkovyskii.

Of his student years at university, Gol'dberg recalls:

"At the university, there were Professors L. I. Volkovyskii and I. G. Sokolov who had the greatest influence on me. This relates to both to their mathematical influence and their civic position. It must be said that in those years a serious "price" could be paid for such a brave civic stand. I was a troublesome student and I was twice expelled from the Young Communist Leaugue and from the university but later reinstated. The offenses were of a political nature: 'bourgeois liberalism', 'loss of Young Communist vigilance' and so on."

"During my first and second years [in the University], I took part in a seminar on the book by Pólya and Szegö conducted by Volkovyskii and Sokolov. During my second year the seminar divided. I went to Sokolov and began to be interested in the constructive theory of functions. I enjoyed this activity; at that time Natanson's clearly written book [English translation: I. P. Natanson, Constructive Function Theory, Frederick Ungar Publishing Co., New York, 1964, - the authors] had appeared. From my fellow students who went to study under Volkovyskii I learned that their fundamental text was R. Nevanlinna's monograph "Eindeutige analytische Funktionen" of which the only copy at Lvov was Volkovyskii's [the Russian translation made by Volkovyskii was printed in the extremely hard year 1941, it was indeed rare everywhere in the Soviet Union - the authors]. To study this book 
one had to go to his apartment at prescribed times. Nevanlinna's book was difficult even for senior students and even worse for second year students who still not studied complex analysis. Having heard their complaints, I thanked my lucky stars that I had distanced myself from that accursed book. But here Chance intervened. Looking through the catalogue of the Lvov Regional Library I saw a card for Nevanlinna's book in the section "Analytic geometry". I could not put the treasure down, ordered the book and defected to the Volkovyskii camp."

Volkovyskii was an analyst working in various fields of complex analysis (quasiconformal mappings, Riemann surfaces). Among his students, besides Gol'dberg, were Pavel Belinskii, Ivan Danilyuk, David Potyagailo, Ivan Pesin, Yurii Rodin, Yurii Trohimchuk. He suggested Gol' dberg to amplify the results known at the time related to the inverse problem of the value distribution theory. It is worth mentioning that the result obtained by Gol'dberg in his Master thesis remained the best achievement in the subject for more than 20 years.

Having graduated (with the Soviet equivalent of MS degree) from Lvov University in 1952, Gol'dberg got a position of secondary school teacher of Mathematics and Physics in a small obscure village Zabolotsy (Swampy) in Galicia. He never was a PhD student of any university since this was almost impossible for a Jew in the Ukraine at that time.

After Stalin's death in 1953, Gol'dberg did apply to enter Lvov University as a PhD student. In the Soviet Union the applicant had to enclose with his application a letter written on his behalf by the authorities and the local Communist Party Committee from his place of work. One sentence from such a letter on Gol'dberg's behalf became famous; it said: "( $\mathrm{He})$ does not drink; even does not drink at all'. Nevertheless, Gol'dberg's application was rejected. By official rules, it was possible to pass the exams and then to submit a PhD thesis for defense without being enrolled as a student. This circumvention was used by Gol'dberg. With a teaching load of more than 30 hours per week and additional official duties, he worked on his Candidate thesis (analogue of PhD thesis) devoted to a subject which he chose. One of results of the thesis was his famous result on the existence of meromorphic functions of finite order with infinitely many deficient values. 
In 1955 he submitted his thesis to Lvov University. The official opponent of the thesis was Boris Ya. Levin who highly praised Gol'dberg's accomplishments. This began the strong scientific contacts and personal friendship between Gol'dberg and Kharkov scholars which continue to this day.

The successful defense of the thesis in 1955 made it possible for Gol'dberg to get a position of Docent at the just organized Uzhgorod University. In those years Uzhgorod was a small provincial town on the Western border of the Soviet Union, not very far from Lvov. In 1963, Gol'dberg received the same position in the much higher regarded Lvov University, and in 1965 completed his Doctoral thesis, a fundamental opus more than 600 pages. One of main achievements of his Doctoral thesis was the theory of integration with respect to a semi-additive measure, with numerous applications to estimates of entire functions. Its successful defense at Kharkov University made it possible for him to claim for a position of Professor at Lvov University. He got this position in 1968 and still occupies it at present in spite of many offers to move.*

In $1965 \mathrm{Gol}$ 'dberg began his seminar at the Mathematics Department of Lvov University, on Tuesdays for two hours. Almost all results of Lvov mathematicians related to the theory of entire and meromorphic functions were thoroughly considered and discussed at the seminar.

Gol'dberg maintains strong scientific ties with many mathematicians. In addition to numerous connections with Kharkov scholars, Gol'dberg kept his ties with late Nikolai Govorov, with many mathematicians from Erevan, Krasnoyarsk, Novosibirsk, Ufa, Vilnius. Many of them used to come to Lvov to talk at his seminar.

With many of his colleagues Gol'dberg keeps intensive correspondence. Besides mathematics, his letters contain concise reports on current economical and political developments, local news, short reminiscences, interesting historical remarks, everything flavored with a wonderful humor. Gol'dberg's talent as a remarkable storyteller goes hand in hand with the literary quality of his letters.

In 1964 Gol' dberg married Basya B. Lekhtman. They have three sons, Alexander, Mikhael and Victor. Gol'dberg brings up a son, Mark, from a previous marriage.

\footnotetext{
* In summer 1997 Gol'dberg moved to Israel and currently has a position in Bar Ilan
} University. 


\section{GOL'DBERG'S MATHEMATICAL WORK'}

\section{Meromorphic Functions}

One of the fundamental achievements in Analysis in the first part of this century was the theory of value distribution of meromorphic functions created by Rolf Nevanlinna [66], [68]. This theory studies asymptotic properties of meromorphic functions, with special emphasis on the asymptotic distribution of their $a$-points. From its first days, the Nevanlinna theory displayed internal depth, coherence and beauty. In the same time, it gave a birth to a series of very difficult analytical problems, most of them already formulated in [66], [68]. For many years, these "inner problems" of the theory were in the focus of attention of analysts. Now, most of these problems have been solved (see [15]). This subject was Gol'dberg's primary interest, and his work constituted a fundamental contribution to their solutions.

We consider functions $f(z)$ meromorphic in the whole complex plane. Let $n(r, a)=n(r, a, f)$ be the number of $a$-points of $f(z)$ in the disc ||$z \mid \leq r\}$ counted with multiplicities, and let $n_{1}(r, a)$ be the number of multiple $a$-points of $f(z)$ in $\{|z| \leq r\}$; i.e. each root of the equation $f(z)=a$ of multiplicity $p$ is counted $p-1$ times. Let $d \omega$ be the area element on the Riemann sphere, normalized so that the total area of the sphere equals one. Then

$$
A(r, f)=\iint_{\hat{C}} n(r, a) d \omega(a)
$$

is the spherical area of the $f$-image of the disk $\{|z| \leq r\}$, counting multiplicity of covering. For many reasons, it is more convenient to use

\footnotetext{
'The authors have neither intension nor space to cover here all aspects of Gol'dberg's mathermatical activity. In particular, important Gol'dberg's work in entire functions of several variables [G12, G14, G18, G26] (and paper [G13] with applications to entire solutions of non-linear partial differential equations), in polyanalytic functions [G20, G79, $\mathrm{G} 80, \mathrm{G} 82]$, in the asymptotic behavior of conformal mappings [G41, G51, G77, G93, G145], and the uniqueness properies of rational and algebraic functions [G135, G138, G147, G149] are outside this survey. Here and in what follows. the reference [G n] pertains to the $n$-th number in the list of Gol'dberg's publications given below.
} 
the integrated characteristics

$$
\begin{aligned}
& N(r, a)=N(r, a, f)=\int_{0}^{r} \frac{n(t, a)-n(t, 0)}{t} d t+n(0, a) \log r, \\
& N_{1}(r, a)=N_{1}(r, a, f)=\int_{0}^{r} \frac{n_{1}(t, a)-n_{1}(t, 0)}{t} d t+n_{1}(0, a) \log r, \\
& T(r, f)=\int_{0}^{r} \frac{A(t, f)}{t} d t=\iint_{\hat{C}} N(r, a) d \omega(a) ;
\end{aligned}
$$

The last quantity is called the Nevanlinna characteristic of $f(z)$.

The Nevanlinna proximity function

$$
\begin{aligned}
m(r, f) & =m(r, \infty, f)=\frac{1}{2 \pi} \int_{0}^{2 \pi} \log ^{+}\left|f\left(r e^{i \theta}\right)\right| d \theta, \\
m(r, a, f) & =m\left(r, \infty, \frac{1}{f-a}\right), \quad a \neq \infty,
\end{aligned}
$$

measures how close on the average $f$ is to $a$. The order and the lower order are defined as

$$
\rho=\limsup _{r \rightarrow \infty} \frac{\log T(r, f)}{\log r}, \quad \lambda=\liminf _{r \rightarrow \infty} \frac{\log T(r, f)}{\log r} .
$$

For entire functions, this definition coincides with the usual one in terms of the maximum modulus. Nevanlinna's two fundamental theorems follow:

for every $a \in \hat{C}$

$$
m(r, a, f)+N(r, a, f)=T(r, f)+O(1), \quad r \rightarrow \infty,
$$

and

if $\left\{a_{1}, \ldots, a_{q}\right\} \subset \hat{C}$, then

$$
\sum_{k=1}^{q} m\left(r, a_{k}, f\right)+N_{1}(r, f) \leq 2 T(r, f)+Q(r, f)
$$

where the remainder term

$$
Q(r, f)=\sum_{k=1}^{q} m\left(r, f^{\prime} /\left(f-a_{k}\right)\right)+O(1)=o(T(r, f))
$$


as $r \rightarrow \infty$ outside an exceptional set of $r$ of finite measure, and

$N_{1}(r, f)=\sum_{a \in \hat{C}} N_{1}(r, a, f)=N\left(r, 0, f^{\prime}\right)+2 N(r, \infty, f)-N\left(r, \infty, f^{\prime}\right)$.

The first fundamental theorem (N1) is an immediate corollary of the Poisson-Jensen formula. However, the second fundamental theorem (N2) lies essentially deeper; its original proof uses Nevanlinna's lemma of the logarithmic derivative:

$$
m\left(r, f^{\prime} / f\right)=O(\log T(r, f)+\log r)
$$

again, as $r$ tends to infinity outside a set of $r$ 's of finite measure. For meromorphic functions of finite order (N3) holds without an exceptional set.

The Nevanlinna deficiency

$$
\delta(a, f)=\liminf _{r \rightarrow \infty} \frac{m(r, a, f)}{T(r, f)}=1-\limsup _{r \rightarrow \infty} \frac{N(r, a, f)}{T(r, f)}
$$

characterizes exceptional behaviour of a-points of $f(z)$. If $\delta(a, f)>0$, then $a$ is a deficient value of $f ; D_{N}(f)=\{a: \delta(a, f)>0\}$ is a set of all deficient values of $f$. The quantity

$$
\epsilon(a, f)=\limsup _{r \rightarrow \infty} \frac{N_{1}(r, a, f)}{T(r, f)}
$$

is called the ramification index of $f(z)$ at $a$. The first fundamental theorem (N1) yields

$$
0 \leq \delta(a, f)+\epsilon(a, f) \leq 1
$$

and the second $(\mathrm{N} 2)$ implies the deficiency relation

$$
\sum_{a \in \hat{C}}\{\delta(a, f)+\epsilon(a, f)\} \leq 2 .
$$

\subsection{The first result: inverse problem of value distribution theory}

Gol'dberg's first result [G1] (see also [G57, Chapter VII]) concerns the inverse problem of value distribution theory. Here is a general formulation of this problem which is due to $R$. Nevanlinna. 
Given a finite or infinite sequence of points $\left\{a_{k}\right\} \subset \hat{C}$ and corresponding nonnegative numbers $\delta\left(a_{k}\right), \epsilon\left(a_{k}\right)$ such that

$$
\begin{gathered}
0<\delta\left(a_{k}\right)+\epsilon\left(a_{k}\right) \leq 1, \quad k=1,2, \ldots, \\
\sum_{k}\left\{\delta\left(a_{k}\right)+\epsilon\left(a_{k}\right)\right\} \leq 2 .
\end{gathered}
$$

Find a meromorphic function with assigned deficiencies $\delta\left(a_{k}\right)$ and indices $\epsilon\left(a_{k}\right)$ at every $a_{k}$ and without other deficiencies and indices.

In 1932, R. Nevanlinna [67] solved this problem under the additional assumptions that

(i) the number of deficient values is finite,

(ii) all deficiencies are rational numbers,

(iii) $\sum_{k} \delta\left(a_{k}\right)=2$ (and hence all indices of multiplicity are zero).

The associated meromorphic function was constructed by Nevanlinna by means of a special class of Riemann surfaces which he introduced, the surfaces with a finite number of logarithmic branch points and no other singularities. Further progress was made in 1949 by Le-Van-Thiem [48], [49]. Making use of surfaces with periodic ends introduced by Ullrich in 1936 [82] and Wittich's ideas of using quasiconformal mappings in value distribution theory [91], [92], Le-Van-Thiem solved the inverse problem under the following assumptions:

(i) the number of deficient and ramified values is finite,

(ii) all deficiencies and indices are rational numbers,

(iii) if $\epsilon\left(a_{k}\right)>0$, then $\delta\left(a_{k}\right)+\epsilon\left(a_{k}\right)<1$,

(iv) $\sum_{k} \delta\left(a_{k}\right)+\epsilon\left(a_{k}\right)=2$.

In fact, assumption (iv) is easy to drop.

Continuing these researches, Gol'dberg introduced a wider class of Riemann surfaces, those with almost periodic ends (see Part 2 of this survey) and carefully investigated the asymptotic behaviour of the corresponding mapping functions. It allowed him to solve the inverse problem under the assumptions:

(i) the number of deficient values is finite (however, the number of indices might be infinite),

(ii) $\sum_{k} \delta\left(a_{k}\right)<2$.

The functions solving the inverse problem under these assumptions were of finite order. It was proved much later by Drasin [14] that functions of finite order with $\sum \delta(a)=2$ must have rational deficiencies. So 
Gol'dberg's result, combined with Nevanlinna result mentioned above gives the complete solution of the inverse problem for functions of finite order with finitely many deficiencies. On the other hand, when the number of pre-assigned deficient values is infinite, deficiencies satisfy one additional relation in addition to (N4) and (N5), namely the sum of cubic roots of deficiencies is convergent (Weitsman [89]), so Gol'dberg's result is the best possible in some sense: none of the assumptions (i) or (ii) can be removed if one wants a function to have finite order.

In 1974, Drasin [13] completely solved the Nevanlinna's inverse problem. As was just explained, the function which solves the problem has to have infinite order. In 1986, Eremenko [24] partially solved the finiteorder variant of the inverse problem with infinitely many deficiencies but his technique does not permit to prescribe indices. Gol'dberg's construction remains one of the main ingredients in Eremenko's example.

In fact, the inverse problem for functions of finite order is at least of the same importance, but much harder than the unrestricted inverse problem. (One of the reasons why one may prefer to restrict with functions of finite order is explained below, in section 1.3.) Here is how Gol'dberg formulated it in a conversation with one of us:

For every given $\rho>0$, describe the set of functions $\delta: \bar{C} \rightarrow[0,1]$ with the property that there exists a meromorphic function $f$ of order $\rho$ for which $\delta(a, f)=\delta(a), a \in \bar{C}$.

Gol'dberg's technique and its extensions in [16] and [24], probably, remain the only tool available at present to attack this problem.

\subsection{Structure of the set of deficient values}

The Nevanlinna deficiency relation (N5) implies that the set of deficient values $D_{N}(f)$ of a meromorphic function $f$ is at most countable. The fundamental question whether there exist meromorphic functions (and, moreover, functions of finite order) with an infinite set of deficient values remained open till 1954. Until that year, the best result (due to R. Nevanlinna [66, p.91]), stated that a meromorphic function $f$ of a prescribed positive order may have an arbitrary large (however, finite) set $D_{N}(f)$; in addition, functions of zero order can have at most one deficient value (Valiron [85]). 
In 1954, Gol'dberg [G4] constructed an example of meromorphic functions of order one with infinite sets of deficiencies. Moreover, he proved that, given an arbitrary and at most countable set $M \subset \hat{C}$, there exists a meromorphic function $f$ of order one such that $M \subset D_{N}(f)$. In 1959, modifying this construction, Gol'dberg proved [G17]

THEOREM 1. Given $\rho, 0<\rho \leq \infty$, and given an arbitrary, at most countable, subset $M$ of the Riemann sphere, there exists a meromorphic function of order $\rho$ whose set of deficient values coincides with $M$.

Together with the above-mentioned result of Valiron, this theorem gives the full description of the structure of the set of deficient values of meromorphic functions of arbitrary order $\rho, 0 \leq \rho \leq+\infty$ : it may be an arbitrarily given an at most countable set if $0<\rho \leq+\infty$, and it contains at most one (arbitrary) point if $\rho=0$.

Hayman [36, p.81] describes the idea of the Gol'dberg construction as follows:

"If

$$
f(z)=\frac{\sum a_{m} \varphi_{m}(z)}{\varphi(z)},
$$

and if $\varphi_{m}$ and $\varphi$ are both large and nearly equal on certain sets $F_{m}$ which are disjoint for different $m$ while $\varphi$ is small outside $F_{m}$, then $f(z) \approx a_{m}$ on $F_{m}$."

Hayman [36, Chapter 4] (see also [G57, Chapter IV]) showed a simpler way to realize this principle and gave a shorter proof of Gol'dberg's theorem.

The idea of Gol'dberg's construction has been used many times in various situations. An impressive result obtained with its help is the result by Fuchs and Hayman [31] (see also [36, Chapter 4], [G57, Chapter IV]) on the existence of entire function of infinite order with not only preassigned set of deficient values but also with pre-assigned values of deficiencies. This result shows that Gol'dberg's construction can be used for solution of the inverse problem of value distribution theory. Perhaps, it may produce another solution of the inverse problem for meromorphic functions which will work as well for entire holomorphic curves in projective space where geometric methods apparently do not work.

The question on the structure of the set of deficient values for entire functions of finite order was solved by Arakelyan [4] (the full exposition 
appeared in [5], see also [G57, Chapter 4, §5]) and Eremenko [23]. Making use of methods of approximation theory, Arakelyan constructed entire functions of finite order with an infinite set of deficient values. He proved that, given an at most countable set $M \subset C$ and given $\rho>1 / 2$, there is an entire function $f(z)$ of order $\rho$ such that $M \subset D_{N}(f)$. Combining the technique of limit sets of subharmonic functions with the technique of quasiconformal deformations, Eremenko produced another construction of entire functions of finite order with infinite set of deficient values. In his construction the whole set of deficient values is pre-assigned: $M=D_{N}(f)$. We recall, that the Wiman-Valiron $\cos \pi \rho$ theorem implies that entire functions of order $\rho \leq 1 / 2$ cannot have finite deficient values.

\subsection{Invariance of the deficiencies}

One may hope that reasonable quantities characterizing asymptotic behaviour of a meromorphic function $f(z)$ depend only on the geometric structure of the associated Riemann surface $F$ (its precise definition will be given in the beginning of Part 2). Since both $f(z)$ and $f_{h}(z)=$ $f(z+h)$ map $C$ conformally on $F$ one may hope that $\delta(a, f)=\delta\left(a, f_{h}\right)$. However, in 1947, Dugue observed [19] that for the function

$$
f(z)=\frac{\exp \left[2 \pi i e^{2}\right]-1}{\exp \left[2 \pi i e^{z}\right]+1}
$$

$\delta\left(0, f_{h}\right)=e^{-h}\left(e^{h}+e^{-h}\right)^{-1}$ and $\delta\left(\infty, f_{h}\right)=e^{h}\left(e^{h}+e^{-h}\right)^{-1}$. In 1953, Hayman [35] has constructed an example of an entire function of infinite order such that $\delta(0, f) \neq \delta\left(0, f_{h}\right)$ for any $h \neq 0$. In the opposite direction, in 1947, Valiron [84] (see also [G57, Chapter IV]) showed that if for meromorphic function $f(z)$

$$
\rho-\lambda<1,
$$

where $\rho$ and $\lambda$ are respectively order and lower order of $f$, then all deficient values are invariant with respect to the shift of the origin. The questions whether the Valiron condition (1) is the sharp one and even whether there exist meromorphic functions of finite order with noninvariant deficiencies remained open when Gol'dberg began his work.

In 1954, he gave complete answers to the both questions [G2]. For each $\rho$ and $\lambda$ such that $\rho-\lambda \geq 1$, there is a meromorphic function of 
order $\rho$ and lower order $\lambda$ with non-invariant deficiencies. Thus, (1) is the best possible. Gol'dberg's original construction [G2] used quasiconformal mappings. Later, due to remark made by Levin at the defence of Gol'dberg's PhD Thesis, this construction was greatly simplified in [G23] (see also [G57, Chapter IV]).

The first example of an entire function of finite (but very large) order with non-invariant deficiencies was constructed by Miles [61]. In [G116], [G117] an entire function of arbitrary order $\rho>5$ such that $0=\delta\left(0, f_{1}\right)<\delta(0, f)$ was constructed. For $\rho \leq 3 / 2$, deficiencies of entire functions of order $\rho$ are invariant because of (1) combined with the $\cos \pi \rho$-theorem. The question thus is still open for $3 / 2<\rho \leq 5$.

In the same paper [G2] (see also [G57, Chapter IV]), Gol'dberg modified Nevanlinna's definition of deficiency for meromorphic functions of finite order in such a way that, keeping all essential properties of Nevanlinna's definition, this modified deficiency is invariant with respect to the shift of the origin. Let $\mathcal{L}$ be a class of all Lebesgue measurable sets $E \subset[1, \infty)$ such that $\operatorname{mes}(E \cap[1, r))=O(\log r), r \rightarrow \infty$, and set

$$
\delta^{*}(a, f)=\sup _{E \in \mathcal{L}} \liminf _{r \rightarrow \infty, r \notin E} \frac{m(r, a)}{T(r, f)} .
$$

It is readily seen that $\delta(a, f) \leq \delta^{*}(a, f), 0 \leq \delta^{*}(a, f) \leq 1$. The second fundamental theorem (N2) easily implies that $\sum_{a} \delta^{*}(a, f) \leq 2$. It turns out that, for meromorphic functions of finite order, $\delta^{*}(a, f)$ does not depend upon the choice of the origin: $\delta^{*}\left(a, f_{h}\right)=\delta^{*}(a, f)$. It is worth mentioning that for many classes of meromorphic functions of finite order $\delta^{*}(a)=\delta(a)$. This is the case if the limit exists in the definition of $\delta(a, f)$ or if Valiron's condition (1) is fulfilled.

\subsection{Non-asymptotic deficient values}

Another shortcoming of the notion of deficiency is that there is no simple connection between deficient values and asymptotic values (or transcendental singularities of the associated Riemann surface $F$ ). During the first decade after the creation of the Nevanlinna theory it was regarded as plausible that all deficient values are asymptotic values [68]. After the examples of Teichmüller [78] and Mme Schwartz [76], it turned out that this is not true. In 1953, Hayman [35] has constructed an entire function of infinite order with a non-asymptotic deficient value. This left open 
whether deficient values of an entire functions of finite order must be asymptotic. Since by the Denjoy-Carleman-Ahlfors theorem the number of asymptotic values of entire function of order $\rho$ cannot exceed $2 \rho$, the affirmative answer to this question would yield an old conjecture of Nevanlinna on the finiteness of the number of deficient values for entire functions of finite order (we have already seen in 1.2 that this conjecture is false).

In 1957, Gol'dberg [G8] showed that for every $\rho>1$ there is an entire function of order $\rho$ with a non-asymptotic deficient value, and thus made the Nevanlinna conjecture essentially less plausible.

In all above-mentioned examples of functions with non-asymptotic values, deficiencies were strictly less than one. The question whether there exist functions with non-asymptotic deficient values with the maximal value of the deficiency $\delta(a, f)=1$ remained open. Gol'dberg constructed the first examples of this type. In 1966, he showed [G49] (see also [G57, Chapter V]) that for every $\rho, 1<\rho \leq+\infty$, there is a meromorphic function of order $\rho$ with a non-asymptotic deficient value $a$ such that $\delta(a)=1$. In 1978, Hayman [37] obtained the final result in this direction: for any function $\psi(r)$ increasing to $+\infty$, there is a meromorphic function $f$ such that $T(r, f)=O\left(\psi(r) \log ^{2} r\right), r \rightarrow \infty$ with a non-asymptotic deficient value $a$ such that $\delta(a, f)=1$. On the other hand, in 1966, Anderson and Clunie proved [2] that if $T(r, f)=$ $O\left(\log ^{2} r\right), r \rightarrow \infty$, then each deficient value must be asymptotic.

It follows from the results of Edrei and Fuchs [20] that if $f$ is an entire function of finite order and $\delta(a, f)=1$, then $a$ is an asymptotic value of $f$. It turns out that for functions of infinite order this is not true: in 1967, Gol'dberg [G52] (see also [G57, Chapter V]) constructed an entire function $f(z)$ of infinite order with a non-asymptotic deficient value $a$ with $\delta(a, f)=1$.

\subsection{The lemma of the logarithmic derivative}

If a meromorphic function $f$ tends rapidly to a value $a \in C$, then it would be natural to assume that $f^{\prime}$ tends at least with the same rate to zero. Since $f^{\prime}=f \cdot\left(f^{\prime} / f\right)$, to justify this assumption one needs a good upper bound for the logarithmic derivative $f^{\prime} / f$. Such a bound is given by the lemma of the logarithmic derivative (see (N3)) which plays an indispensable role in the theory of meromorphic functions and 
in the analytic theory of differential equations. More exactly, Nevanlinna proved that, for every $R>r$,

$$
\begin{aligned}
m\left(r, f^{\prime} / f\right)< & 4 \log ^{+} T(R, f)+3 \log ^{+} \frac{1}{\left|c_{s}\right|}+2 \log ^{+} \frac{1}{r} \\
& +4 \log ^{+} R+3 \log ^{+} \frac{1}{R-r}
\end{aligned}
$$

where $c_{s}$ is the first non-vanishing coefficient in the power expansion of $f$ at the origin. Applying arguments conceming growth of monotone functions (so called Borel-Nevanlinna lemma [66], [68], [G57, Theorem 1.2, Chapter III]), Nevanlinna deduced from (2) that

$$
m\left(r, f^{\prime} / f\right)<12+8 \log ^{+} T(r, f)+3 \log ^{+} \frac{1}{\left|c_{s}\right|}+4 \log ^{+} r
$$

with the exception of a set of values $r$ of finite measure, which is the key ingredient in Nevanlinna's proof of (N3). If $f(z)$ is of finite order, then (2) directly yields (N3) without exceptional intervals.

Estimates (2) and (3) were refined in a number of papers, among them were [69], [45] and many others. Making use of ideas from [43] and [69], Gol'dberg and Grinshtein found in [G78] a very sharp version of estimate (2). Namely, they proved that, if $f(0)=1$, then

$$
m\left(r, f^{\prime} / f\right)<\log ^{+}\left\{\frac{T(R, f)}{r}\left(1-\frac{r}{R}\right)^{-1}\right\}+5.8501 .
$$

As shown in [G78] this estimate turns out to be sharp in several ways.

Paper [G78] was an isolated result in the subject until the end of the last decade when formal analogies between value distribution theory and number theory (problems of Diophantine approximation) drew the attention of Ch. Osgood [70], Lang [47] and Vojta [87]; see [87] for a "dictionary" between these two subjects. Translating his old conjecture on rational approximation of irrational numbers to value distribution theory, Lang asked in [47] for precise estimate for the remainder term $Q(r)$ in the second fundamental theorem (N2) and conjectured purely for formal reasons that for an arbitrary positive continuous function $\phi(t)$ such that $\phi(t) / t$ increases and $\int^{\infty} \phi^{-1}(t) d t<\infty$,

$$
Q(r) \leq \log \phi(T(r, f))
$$


holds outside an exceptional set of finite measure. It turned out, that the best possible error term in the lemma of the logarithmic derivative is almost the same as the best possible error term in the second fundamental theorem. Namely, adding to (4) a growth lemma of BorelNevanlinna type, Miles proved [63] estimate (5) for $m\left(r, f^{\prime} / f\right)$ instead of $Q(r)$. Furthermore, given a function $\phi(t)$ such that $\phi(t) / t$ increases and $\int^{\infty} \phi^{-1}(t) d t=+\infty$, he constructed an entire function $f$ such that

$$
m\left(r, f^{\prime} / f\right) \geq \log \phi(T(r, f))
$$

outside a set of finite measure. Approximately at the same time and also depending heavily on (4), Hinkannen [41] proved Lang's conjecture (5). In fact, in [41] a sharper result was proved.

Another contribution by Gol'dberg to our understanding of the logarithmic derivative [G75] deals with meromorphic functions in the halfplane $C_{+}=\{\Im z \geq 0\}$. Let $f(z)$ be a meromorphic function in $C_{+}$. R. Nevanlinna [65] (see also [G57]) has introduced the following characteristics: the counterparts of the proximity function

$A(r, f)=A(r, \infty, f)=\frac{1}{\pi} \int_{1}^{r}\left(\frac{1}{t^{2}}-\frac{1}{r^{2}}\right)\left[\log ^{+}|f(t)|+\log ^{+}|f(-t)|\right] d t$

and

$$
B(r, f)=B(r, \infty, f)=\frac{2}{\pi r} \int_{0}^{\pi} \log ^{+}\left|f\left(r e^{i \theta}\right)\right| \sin \theta d \theta,
$$

the counting function

$$
c(r, f)=c(r, \infty, f)=\sum_{\{b: f(b)=\infty, 1<|b| \leq r\}} \sin (\arg b),
$$

the integrated counting function

$$
C(r, f)=C(r, \infty, f)=2 \int_{1}^{r} c(t, f)\left(\frac{1}{t^{2}}+\frac{1}{r^{2}}\right) d t,
$$

and the analog of the characteristic function

$$
S(r, f)=A(r, f)+B(r, f)+C(r, f) .
$$

In these notations a direct counterpart of the lemma of the logarithmic derivative would be the relation

$$
A\left(r, f^{\prime} / f\right)+B\left(r, f^{\prime} / f\right)=o(S(r, f))
$$


when $r$ tends to infinity outside an exceptional set, and would imply an analog of the second fundamental theorem (N2). Nevanlinna claimed [65] that such an analog is in general valid but at that time did not wish to go into a detailed investigation, and so confined himself to the case when the function $f(z)$ is meromorphic in the whole complex plane and has finite order. Under these restrictions he proved that $A\left(r, f^{\prime} / f\right)+B\left(r, f^{\prime} / f\right)=$ $O(1)$. This result was generalized by Dufresnoy [17] and Ostrovskii [71] (see also [G57, Chapter III]). However, (6) remained unconfirmed until 1974 when Gol'dberg has constructed an unexpected counterexample. He showed that, for any function $\Phi(r) \rightarrow+\infty, r \rightarrow \infty$, there is an entire function $f(z)$ such that $S(r, f) \equiv 0$ but $A\left(r, f^{\prime} / f\right) / \Phi(r) \rightarrow \infty$. Thus (6) is not valid and, moreover, it cannot be even modified in an appropriate way.

The analog of the second fundamental theorem $(\mathrm{N} 2)$ for meromorphic functions in half-plane remains unproved up to now; moreover, there is no supporting evidence. For a weaker version of it see [G57, Chapter III].

In a recent work Grishin [34] proposed the function $B(r, f)$ as the "right" proximity function and $A(r, f)+C(r, f)$ as the "right" counting function. Such a choice is rather natural for the theory of analytic functions in the unit disc or in the upper half-plane. Then counterparts of the lemma of the logarithmic derivative (N3) and of the second fundamental theorem (N2) (in a somewhat weaker form: without a term responsible for multiple points) turn out to be true. However, to prove the analog of (N2) Grishin relies upon the potential-theoretic arguments from [25].

\subsection{Meromorphic functions with separated zeros and poles}

In 1960, Gol'dberg introduced [G19] (see also [G57, Chapter VI]) the class of meromorphic functions with $(p, \eta)$-separated zeros and poles, $p \in N, 0 \leq \eta<\pi /(2 p)$. It consists of the functions $f$ such that, for some $\phi, 0 \leq \phi<2 \pi$, all zeros of $f$ are in the union of angles

$$
\bigcup_{j=0}^{p-1}\{z:|\arg z-\phi-(2 j \pi) / p| \leq \eta\}
$$

and all poles of $f(z)$ are in the union of angles

$$
\bigcup_{j=0}^{p-1}\{z:|\arg z-\phi-(2 j+1) \pi / p| \leq \eta\} .
$$


He proved in [G19] the result which for the first glance is rather unexpected:

THEOREM 2. If zeros and poles of a meromorphic function $f$ are $(p, \eta)$ separated, then there exists finite or infinite limit

$$
\lim _{r \rightarrow \infty} \frac{T(r, f)}{r^{p}}
$$

In particular, (7) implies that if the lower order $\lambda<p$, then the order $\rho \leq p$.

Theorem 2 has interesting consequences for meromorphic functions with radially distributed zeros and poles. For simplicity, we restrict here ourselves by the most interesting case of entire functions. Let $f(z)$ be an entire function with zeros on the system of rays

$$
D=D\left(\alpha_{1}, \ldots, \alpha_{n}\right)=\bigcup_{j=1}^{n}\left\{z: z=r e^{i \alpha_{j}}\right\}
$$

and let $G_{p}$ be the convex hull of the set $\left\{e^{i \alpha_{1}}, \ldots, e^{i \alpha_{n}}\right\}, p \in N$. If the origin does not belong to $G_{p}$, then zeros of $f(z)$ are $(p, \eta)$-separated with some positive $\eta$. Thus Theorem 2 implies

COROLLARY If, for some $p \in N$, the origin does not belong to $G_{p}$, then either $\rho \leq p$, or $\lambda \geq p$ (i.e., $\lambda<p<\rho$ can never occur).

For example, if $n=1$, then, for every $p \in N$, the origin does not belong to $G_{p}$ and we conclude that for entire functions with zeros on a ray $\rho-\lambda \leq 1$. Under the a priori assumption $\rho<\infty$, this was first proved by Edrei and Fuchs [20]. If $n=2$, then, for every $p \in N$, at least for one of numbers $p, p+1$ the origin does not belong to $G_{p}$. Therefore, for entire functions with zeros on two rays $\rho-\lambda \leq 2$. These facts were rediscovered by several authors, see e.g. [77]. For $n=3$, Gleizer [32] deduced from the Corollary that $\rho \leq 3([\lambda]+1)$, and that this relation is sharp. In the general case, Theorem 2 and its Corollary show an interesting interplay with number theory: the growth of entire function with radially distributed zeros depends essentially on the arithmetic nature of arguments of zeros.

Gleizer [32] and Miles [62] supplemented this Corollary by the converse statement: given a system of rays $D$ and positive integers $p_{-}, p_{+}$, $p_{-} \leq p_{+}$, such that, for every $p=p_{-}, p_{-}+1, \ldots, p_{+}$, the origin 
belongs to $G_{p}$, there exists an entire function $f(z)$ with zeros on $D$ such that $\lambda<p_{-} \leq p_{+}<\rho$. Miles' result was slightly weaker; however he considered a more general case of meromorphic functions. He also proved that under certain additional assumptions the assertion of Corollary remains true if the origin belongs to the boundary of $G_{p}$.

In [G25], Gol'dberg proved another result about meromorphic functions with separated zeros and poles. Let

$$
\kappa(f)=\limsup _{r \rightarrow \infty} \frac{N(r, 0, f)+N(r, \infty, f)}{T(r, f)} .
$$

THEOREM 3. Let $f(z)$ be a meromorphic function with $(p, \eta)$-separated zeros and poles. If

$$
0<\lim _{r \rightarrow \infty} \frac{T(r, f)}{r^{p}}<\infty
$$

then $\kappa(f)=0$. If

$$
\lim _{r \rightarrow \infty} \frac{T(r, f)}{r^{p}}=\infty
$$

then

$$
\kappa(f) \leq \frac{2}{1+\cos p \eta}
$$

See [G57, Chapter VI] for the proofs of Theorems 2 and 3, as well for related results by Edrei, Fuchs, and Hellerstein. For further developments, see the papers by Hellersten and Shea [39] and Miles [60].

\subsection{Teichmüller's conjecture and an inequality for functions convex with respect to logarithm}

In 1905, Wiman [90] proved that for every entire function $f(z)$ of order less than $1 / 2$ there is a sequence of circles $\left\{|z|=r_{n}\right\}, r_{n} \rightarrow \infty$, on which $f(z)$ tends to $\infty$ uniformly with respect to $\arg z$; i.e.

$$
\limsup _{r \rightarrow \infty} \mu(r, f)=\infty
$$

where $\mu(r, f)=\min _{|z|=r}|f(z)|$. This theorem together with subsequent results by Littlewood and Valiron were the starting point for the whole direction in the theory of entire and meromorphic functions (" $\cos \pi \rho$ theorem and related extremal problems"), see [44], [26], [38]. In 1939, 
Teichmuller [78] conjectured that Wiman's theorem can be extended onto meromorphic functions in the following form: if $f(z)$ is a meromorphic function of order $\rho<1 / 2$, for which

$$
\delta(\infty, f)>1-\cos \pi \rho
$$

then (8) still holds, and proved this conjecture under the additional assumption that all zeros of $f(z)$ are positive and all poles are negative. In the general case, he proved (8) under another assumption which is stronger than (9): $\delta(\infty, f)>(1-\cos \pi \rho) /(1-\kappa \cos \pi \rho)$ where $\kappa$ is a positive numerical constant.

In $[G 4, G 6]$ Gol'dberg proved the Teichmüller conjecture. For this purpose, he established an extremal property of meromorphic functions with positive zeros and negative poles.

THEOREM 4. Let $f(z)$ be a meromorphic function of genus zero,

$$
f(z)=C z^{m} \frac{\prod\left(1-z / a_{k}\right)}{\prod\left(1-z / b_{m}\right)}
$$

and let

$$
\hat{f}(z)=C z^{m} \frac{\prod\left(1-z /\left|a_{k}\right|\right)}{\prod\left(1+z /\left|b_{m}\right|\right)} .
$$

Then

$$
m(r, \infty, f) \leq m(r, \infty, \hat{f}), \quad m(r, 0, f) \leq m(r, 0, \hat{f})
$$

and hence

$$
\delta(\infty, f) \leq \delta(\infty, \hat{f}), \quad \delta(0, f) \leq \delta(0, \hat{f}) .
$$

Since $\mu(r, f) \geq \mu(r, \hat{f})$, the Teichmüller conjecture now follows from the particular case proved in [78]. It is a major unsolved problem, how to extend these inequalities to the case of genus greater then zero.

Theorem 4, in its turn, follows from a new integral inequality for convex functions which was proved in [G6], and then was essentially improved in [G9]:

THEOREM 5. Let $\Phi(y)$ be a function convex with respect to $\log y$, for $0<y<\infty$. Let $y_{\nu}(x), \nu=1,2, \ldots, n$ be even $2 \pi$-periodic functions 
of $x$ which do not decrease on $[0, \pi]$ and satisfy the condition $0<m \leq$ $y_{v}(x) \leq M<\infty$. Then, given arbitrary real numbers $\tau_{v}, v=1,2, \ldots, n$,

$$
\int_{-\pi}^{\pi} \Phi\left\{\prod_{\nu=1}^{n} y_{\nu}\left(x+\tau_{\nu}\right)\right\} d x \leq \int_{-\pi}^{\pi} \Phi\left\{\prod_{\nu=1}^{n} y_{\nu}(x)\right\} d x .
$$

A proof of Theorem 5 may be found in [36, Chapter 4].

Theorem 5 implies an inequality which is more general than (10):

$$
\int_{-\pi}^{\pi} \Phi\left(\left|f\left(r e^{i \theta}\right)\right|\right) d \theta \leq \int_{-\pi}^{\pi} \Phi\left(\left|\hat{f}\left(r e^{i \theta}\right)\right|\right) d \theta
$$

which holds for an arbitrary function $\Phi$ convex with respect to $\log y$.

Theorems 4 and 5 found a number of applications to meromorphic functions of genus zero, see [36, Chapter 4]. Relation (8) was essentially improved in [G30, G57 (Chapter V)] as follows. For every meromorphic function $f(z)$ of lower order $\lambda<1 / 2$, the inequality

$$
\liminf _{r \rightarrow \infty} \frac{\mu(r, f)}{T(r, f)} \geq \frac{\pi \lambda}{\sin \pi \lambda}(\delta(\infty, f)-1+\cos \pi \lambda)
$$

holds. This inequality is sharp.

Due to the *-function introduced and applied by Baernstein [8] (see also [38]), we have now a much more powerful tool for study of extremal problems for meromorphic functions. However, it seems that the area of applications of Theorem 5 is far from exhausted.

\subsection{Exceptional linear combinations of entire functions}

Gol'dberg's contribution to the subject of holomorphic curves started with his account of H. Cartan's Second Main Theorem in [G23]. For many years, this remained almost the only source of information about this theory available to Soviet mathematicians.

His original results about holomorphic curves appear in [G60, G70]. Consider a vector of entire functions $\left(g_{1}, \ldots, g_{p}\right)$. The study of zero distribution of linear combinations

$$
f_{j}=\sum_{k=1}^{p} a_{j k} g_{k}
$$


is a classical subject going back to Borel. A system of linear combinations is called admissible if every $p$ rows of the matrix $\left(a_{j k}\right)$ are linearly independent. If $g_{1}, \ldots, g_{p}$ are linearly independent then at most $p$ linear combinations from any admissible system may have no zeros (Borel) and under the weaker condition that not all $g_{k}$ are proportional the number of linear combinations without zeros is at most $2 p-2$ (Montel).

To formulate the general result obtained by Gol'dberg and Tushkanov in $[G 60, G 70]$ we need the following definitions. Let $U$ be a positive increasing function on $[0, \infty)$ with the property $U(2 r)=O(U(r)), r \rightarrow \infty$. Let $\mathcal{K}_{2}$ be the field of all meromorphic functions satisfying $T(r, f)=$ $O(U(r)), r \rightarrow \infty$, and let $\mathcal{K}_{1}$ be any subfield of $\mathcal{K}_{2}$. Denote by $d_{1}$ and $d_{2}$ the dimensions of the linear spans of $\left\{g_{1}, \ldots, g_{p}\right\}$ over $\mathcal{K}_{1}$ and $\mathcal{K}_{2}$ respectively, so that $d_{1} \geq d_{2}$.

A linear combination (11) with coefficients $a_{j k} \in \mathcal{K}_{1}$ is called exceptional if

$$
N\left(r, 0, f_{j}\right)=o(U(r)), \quad r \rightarrow \infty .
$$

THEOREM 6. If $d_{2} \geq 2$, then the number of exceptional combinations in any admissible system (with coefficients from $\mathcal{K}_{1}$ ) is at most

$$
p+\left[\frac{p-d_{1}}{d_{2}-1}\right]
$$

The modern geometric language speaks of preimages of hyperplanes under a holomorphic curve $F: C \rightarrow P^{n}$, rather than zeros of linear combinations Indeed, a vector of entire functions $\left(g_{1}, \ldots, g_{p}\right)$ defines a holomorphic curve in $P^{n}$ where $n=p-1$. Let us choose $\mathcal{K}_{1}=\mathcal{K}_{2}=C$ in the Gol'dberg-Tushkanov theorem (this corresponds to the choice $U \equiv 1$ ). Thus $d_{2} \geq 2$ means that the curve is not constant and in fact $d:=d_{1}-1$ is the dimension of the smallest linear hull in $P^{n}$ of the image of the curve. Then Theorem 6 implies that a curve may omit at most

$$
\left[\frac{n}{d}\right]+n
$$

hyperplanes, a result of Dufresnoy [18] (see also Green [33]). If $d=n+1$ or $d=1$ we obtain respectively Borel's and Montel's results mentioned above.

Independently, a less precise form of Theorem 6 (as well as several other results in the same spirit) was found by Toda [80] (see also [81]). 
Permitting the coefficients of linear combinations to be meromorphic functions of restricted growth is equivalent to the study of the distribution of preimages of "slowly moving targets" (see, for example, [75]), a subject which now plays increasing role. The result of Gol'dberg and Tushkanov still seems to be the most precise available extension of Picard's theorem of this sort.

It is interesting that in [G70] the authors notice a formal analogy with number theory (the main tool used in the paper, Borel's theorem, has a counterpart in transcendental number theory, Lindemann's theorem on linear independence of exponents of algebraic numbers). This was long before another parallel with number theory discovered by Ch. Osgood, Lang and Vojta [87] started to play so important role in Nevanlinna theory. As Gol'dberg mentioned to the authors, a similar analogy between Borel's and Lindemann's theorems was found by Narasimhan in [64], who re-proved Borel's result, but during the time of preparing [G60, G70] Gol'dberg did not.know about [64].

\subsection{Meromorphic solutions of differential equations}

The study of growth of meromorphic solutions of (non-linear) algebraic differential equations probably began with the work of Valiron (see, for example [83]) and Pólya [73]. Valiron proved that entire solutions of

$$
F\left(y^{\prime}, y, z\right)=0, \quad F \text { a polynomial in } 3 \text { variables, }
$$

have finite order, and Pólya showed that such solutions have positive order. Later J. Malmquist [57] proved that in fact the order of entire solutions of (12) is always a multiple of $1 / 2$.

The first general result on the growth of meromorphic solutions is contained in Gol'dberg's paper [G7]. He proved that the order of a meromorphic solution of (12) is always finite and gave an effective estimate in terms of coefficients. The argument used in this paper is so simple and powerful that it permits estimating the growth of solutions even in the case when $F$ is a polynomial only with respect to $y^{\prime}$ and $y$ having transcendental meromorphic coefficients.

Since the paper [G7] was published, several new proofs of this result were given (including Bergweiler's contribution to this volume) but nevertheless the result remains quite isolated: for algebraic equations of higher order no general results are known about growth of meromorphic 
solutions. It is even not known whether entire solutions of an algebraic differential equation of second order have any a priori growth estimate. Some results for equations of second (and higher) order are contained in works by Bank, Laine, Steinmetz as well as in [G86], see the recent book by Laine [46].

In a joint paper with $A$. Mokhon'ko [G76] the authors extend Gol'dberg's result to the case when solutions are meromorphic in an angular sector.

In [21] the question of possible growth of meromorphic (in $C$ ) solutions of (12) was solved completely: the only possible values of the order are multiples of $1 / 2$ or $1 / 3$. If the order is zero, then $T(r, y) \sim$ $c \log ^{2} r, r \rightarrow \infty$.

\section{Simply Connected Riemann Surfaces}

One of the main problems of the theory of meromorphic functions is the study of connection between asymptotic properties of a meromorphic function and the geometry of the associated Riemann surface. To state the problem precisely we need some definitions.

Let $\sigma$ and $\rho$ be the Euclidean metric in $C$ and the spherical metric in $\hat{C}$ respectively. A meromorphic function $f: D \rightarrow \hat{C}, D \subset C$, can be split into composition of a conformal homeomorphism followed by a local isometry in the following way. Let $f^{*} \rho$ be the pullback of the spherical metric. Then the mapping $\theta(z)=z$ from $D$ equipped with $\sigma$ to $D$ equipped with $f^{*} \rho$ is a conformal isomorphism. The mapping $\pi(z)=f(z)$ from $\dot{D}$ equiped with $f^{*} \rho$ to $\hat{C}$ equipped with $\rho$ is a local isometry. Thus $f=\pi \circ \theta$ has been factored into two parts: the part $\theta$ contains information about distortion and the part $\pi$ contains all topological information about $f$. The mapping $\pi$ may be characterized in pure topological terms: for every point $z_{0} \in D$ there are local coordinates $\zeta$ at $z_{0}$ and $w$ at $f\left(z_{0}\right)$ such that the map $f$ is expressed in these coordinates as $w=\zeta^{n}$, where $n \geq 1$ is an integer. Such maps are called topologically holomorphic. ${ }^{2}$ The points where $\pi$ is not locally homeomorphic are isolated; they are called critical points and their images are called critical values.

\footnotetext{
2 We prefer this name aparently invented by Douady and Hubbard to the classic term "inner in the sense of Stoilov".
} 
Now, assume that $R$ is an abstract surface and a topologically holomorphic map $\pi: R \rightarrow \hat{C}$ is given. Then the conformal structure can be pulled back to $R$ via $\pi$ so that $R$ becomes a Riemann surface. The pair $(R, \pi)$ is called a "Riemann surface spread over the sphere" or "Überlagerungsfläche". If $R$ is open and simply connected, there is a conformal homeomorphism $\theta: D \mapsto R$, where $D$ is the unit disk or the plane $C$. In the first case, the Riemann surface is called hyperbolic and in the second case, parabolic. So $f=\pi \circ \theta$ is a meromorphic function in the unit disk or in the plane, which is almost completely determined by $(R, \pi)$ (up to a conformal automorphism of $D$ ). We call the pair $(R, \pi)$ the Riemann surface associated with $f$ if $f=\pi \circ \theta$ where $\theta$ is a conformal homeomorphism of the unit disk or the plane onto $R$.

The central problem of meromorphic function theory ("ein Hauptproblem der modemen Funktionentheorie") according to Nevanlinna, Ahlfors and Teichmüller is to find out how properties of $f$ are determined by geometric properties of the associated $(R, \pi)$. By geometric properties they usually mean those which can be formulated explicitly in terms of $\pi$. They may include topological properties, such as the number of omitted values, asymptotic values, critical values, or properties formulated in terms of the spherical metric, for example the rate of convergence of critical values to some point. On the other hand when they speak of asymptotic properties of $f$, they usually mean the properties which depend of the exhaustion of $C$ by concentric disks with respect to Euclidean metric; these include order, type, deficiencies and so on.

The first problem, of course, is to determine the conformal type of $R$ ([68], Volkovyskii [88] and Wittich [93]). But assume that it is already known that our Riemann surface has the conformal type of the plane (that is we have a parabolic simply connected Riemann surface $F$ ). Our next topic is how geometric properties of $F$ influence the behavior of the associated meromorphic function.

\subsection{Extension of the Denjoy-Carleman-Ahlfors theorem}

From now on, we consider only parabolic simply connected Riemann surfaces.

Let $F=(R, \pi)$ be a Riemann surface. Let us fix a point $a \in \hat{C}$ and consider the family $\{U(\epsilon)\}_{\epsilon>0}$ of open disks of spherical radius $\epsilon$ 
centered at $a$. For every $\epsilon>0$ choose a component $V(\epsilon) \subset C$ of the preimage $\pi^{-1} U(\epsilon)$ in such a way that $\epsilon_{1} \leq \epsilon_{2}$ implies $V\left(\epsilon_{1}\right) \subset V\left(\epsilon_{2}\right)$. Then there are two possibilities:

a) $\cap_{\epsilon>0} V(\epsilon)=\{$ a point in $C\}$, or

b) $\cap_{\epsilon>0} V(\epsilon)=\emptyset$.

In the latter case we say that our choice $\epsilon \mapsto V(\epsilon)$ defines a singularity of the Riemann surface $F$. Hurwitz was the first who observed that the asymptotic paths of $f$ correspond to the singularities of $F$. Each set $V(\epsilon)$ from our family is called an $\epsilon$-neighborhood of the singularity. A singularity is called direct if for some $\epsilon$ the set $V(\epsilon)$ contains no preimages of the point $a$. Otherwise, it is called indirect. This classification belongs to Iversen. (Gol'dberg in [G6] uses the name "critical point" instead of "singularity".)

One version of the Denjoy-Carleman-Ahlfors Theorem asserts that if a Riemann surface associated to a meromorphic function $f$ has $p \geq 2$ direct singularities then

$$
\liminf _{r \rightarrow \infty} r^{-p / 2} T(r, f)>0
$$

(possibly infinite) [68].

In [G6] Gol'dberg extends this theorem by introducing a wider class of singularities than the direct ones, for which this conclusion still holds. He calls these $K$-points. To define a $K$-point we consider a singularity which is defined by $\epsilon \mapsto V(\epsilon)$. Let $h_{\epsilon}$ be the least harmonic majorant of the subharmonic function $\log \left|\psi_{\epsilon} \circ f\right|$ in $V(\epsilon)$ where $\psi_{\epsilon}$ is the conformal homeomorphism mapping $U(\epsilon)$ onto the unit disk, $\psi_{\epsilon}(a)=0, \epsilon<\epsilon_{0}$. If $h_{\epsilon} \neq 0$ for all $\epsilon<\epsilon_{0}$, we say that $V(\epsilon)$ is a $K$-point. It is evident that a direct singularity is a $K$-point. The Denjoy-Carleman-Ahlfors Theorem extends to $K$-points with the same formulation and essentially the same proof. Then Gol'dberg shows that in the definition of $K$-point the words "for all $\epsilon<\epsilon_{0}$ " can be replaced by "for some sequence $\epsilon \rightarrow 0$ " thus showing that the property of a singularity to be a $K$-point depends only on the structure of the Riemann surface in arbitrary small neighborhoods $V(\epsilon)$ of this singularity.

The most important result of this paper is an explicit geometric criterion of a $K$-point for a special class of Riemann surfaces. This class can be defined in the following way: Let $F=(R, \pi)$, and assume that there are two anti-conformal involutions $\tau_{1}$ and $\tau_{2}$ of $R$ with the properties $\pi \circ \tau_{1}=1 / \bar{\pi}$ and $\pi \circ \tau_{2}=\bar{\pi}$. Furthermore, the preimage of the unit disk 
$\pi^{-1} U$ is connected, the preimage of 0 is pointwise fixed by $\tau_{2}$ and there are exactly two singularities, one over 0 and another over $\infty$. From these assumptions one can deduce that $R$ is of parabolic type and the conformal homeomorphism $\theta: C \rightarrow R$ can be chosen in such a way that $f=\pi \circ \theta$ has the form

$$
f(z)=e^{-\delta z} \prod_{K=0}^{\infty} \frac{z-r_{k}}{z+r_{k}}, \quad \sum_{k} \frac{1}{r_{k}}<\infty, \quad r_{k}>0, \delta \geq 0 .
$$

This function always has growth of at most order one, normal type. It is exactly of order one, normal type, if and only if $\delta>0$.

Long before, Teichmuller considered this class of meromorphic functions and associated Riemann surfaces in [79]. He asked whether one can give a geometric condition on the Riemann surface, which is equivalent to $\delta=0$. This was solved completely in Gol'dberg's paper [G6], where he showed that the singularities over 0 and $\infty$ are $K$-points if and only if $\delta>0$ and gives the following necessary and sufficient condition for these properties in terms of critical values $s_{k}$ of $f$ :

$$
\sum_{k=1}^{\infty} \frac{1}{\inf _{j \geq k}\left\{-\log s_{j}\right\}}<\infty .
$$

This result is equivalent to complete geometric characterization (in the sense we just explained) of Riemann surfaces associated to Blaschke products whose zeros lie on a radius. For more general classes of Riemann surfaces, the problem of a geometric characterization of $K$-points remains unsolved.

Let us explain how this problem can be reduced to a problem about inner functions in the unit disk $U$. Let $V(\epsilon)$ be a neighborhood of a singularity over the point 0 and $\phi_{\epsilon}: U \rightarrow V(\epsilon)$ be a universal covering. Then $g=\psi_{\epsilon} \circ \pi \circ \phi_{\epsilon}$ is an inner function which means that its boundary values exist almost everywhere and belong to the unit circle. Now, the singularity over 0 will be a $K$-point if and only if this inner function $g$ is not a Blaschke product. Another way to say the same is that 0 is not a deficient value in the sense of Lehto $[50,51,52]$. An important special case (which always occurs in the case when $f$ is entire) is when $V(\epsilon)$ is simply connected. If we further assume that $V(\epsilon)$ is a neighborhood of only one singularity (which can be always assumed if $f$ is an entire 
function of finite order) then the corresponding inner function $g$ has only one singular point on the unit circle, and we come to the problem of geometric characterization of Blaschke products in the class of all such inner functions. Gol'dberg's result solves the problem with the additional assumptions that zeros lie on a ray.

We decided to include in this volume a translation of the relevant part of this paper for the following reasons. It is published in a journal which is practically unavailable not only in the West but also in the former Soviet Union. And we feel even today that the main result is important enough to be preserved and documented.

The notion of $K$-singularity was extended by Heins [40] who introduced a kind of multiplicity of a $K$-singularity which he called "harmonic index". Heins further improved the Denjoy-Carleman-Ahlfors theorem by replacing the number $p$ of $K$-singularities by the sum of their harmonic indices. He also proved that the set of projections of direct singularities of a parabolic Riemann surface is at most countable while the set of direct singularities themselves may be uncountable. It is not known whether the set of projections of $K$-points of a parabolic surface is always at most countable.

Geometric characterization of Blaschke products with zeros on a ray in [G6] had a surprising application [G91] in proving an extremal property of very slowly growing meromorphic functions, conjectured by $P$. Barry [10].

THEOREM 7. If $f$ is a meromorphic function of order zero satisfying

$$
\liminf _{r \rightarrow \infty} \frac{N(r, 0, f)+N(r, \infty, f)}{\log ^{2} r} \leq \sigma<\infty,
$$

then

$$
\limsup _{r \rightarrow \infty} \frac{\min _{\theta}\left|f\left(r e^{i \theta}\right)\right|}{\max _{\theta}\left|f\left(r e^{i \theta}\right)\right|} \geq C(\sigma)
$$

where

$$
C(\sigma)=\left(\prod_{n=1}^{\infty} \frac{1-q^{2 n-1}}{1+q^{2 n-1}}\right)^{2}, \quad q=\exp (-1 /(4 \sigma)),
$$

and this estimate is the best possible.

For entire funcitons, a version of this theorem (with $N(r, 0, f)+$ $N(r, \infty, f)$ replaced by $\log M(r, f)$ and without condition of zero order) was obtained later by Fenton using other tools [30]. 


\subsection{Riemann surfaces with finitely many "ends"}

In fact, there are only very narrow classes of Riemann surfaces for which a detailed information about asymptotic behavior of the associated function can be derived from the geometric properties of the surface. Still these classes lead to many interesting examples in meromorphic function theory.

In the late 20-s and early 30-s, F. and R. Nevanlinna, Ahlfors considered Riemann surfaces which have finitely many (logarithmic) singularities and no critical points. It was established that these surfaces are of parabolic type, and in fact very precise statements about associated meromorphic functions $f$ can be made. For example, if there are $p$ singularities then the order of $f$ is $p / 2$, normal type, and deficiency $\delta(a, f)$ is the number of singularities over $a$ divided by $p / 2$. So deficiencies of functions of this class are always rational and add up to 2 . The simplest example is $\exp z$ whose associated Riemann surface (the Riemann surface of logarithm) has two singular points. It was Ahlfors who first applied to the study of such Riemann surfaces a geometric method which was the base of all subsequent generalizations. A rough outline of the method in its present form as described in [93] and [G57, Chapter VII] is the following. One dissects the Riemann surface into simply connected pieces such that conformal map of a plane region onto each piece can be found explicitly. The non relatively compact pieces are usually called ends. For example, a logarithmic end is the Riemann surface associated with the restriction of $L \circ \exp$ to the upper half-plane, where $L$ is a fractional linear transformation. It can be shown by a topological argument that a surface with $p$ singularities and finitely many critical points can be partitioned into $p$ logarithmic ends plus one compact piece.

Once conformal maps of plane regions onto ends are known, the problem is to paste them together. This is usually performed now using quasiconformal mappings. Assume that $R=\cup_{j=0}^{p} \bar{R}_{j}$, where $R_{0}$ is the compact piece; $R_{j}, j=1, \ldots, p$, are the ends and bar means the closure in $R$. Assume further that we have conformal mappings $\theta_{j}: S_{j} \rightarrow R_{j}$, where $S_{j}$ are some angular sectors in the plane whose interiors are disjoint, and their closures cover the plane minus a compact set. Then one tries to replace $\theta_{j}$ by certain non-conformal homeomorphisms $\theta_{j}^{\prime}$ which fit together in the sense that $\theta_{k}^{\prime}(z)=\theta_{j}^{\prime}(z)$ at points $z$ on the common part of the boundaries $\partial S_{k}$ and $\partial S_{j}$. This then defines a homeomorphism $\theta^{\prime}: C \rightarrow R$, namely $\theta(z)=\theta_{j}^{\prime}(z)$ if $z \in S_{j}$. This explicitly 
constructed homeomorphism has any value only if it is in some sense close to conformal, which depends essentially on the choice of the maps $\theta_{j}^{\prime}$. If $\theta^{\prime}$ is quasiconformal then a theorem of Grötzsch guarantees that $R$ is of parabolic type (otherwise, a quasiconformal mapping of the complex plane onto the unit disk would exist).

Sometimes one can say much more, using more careful choice of $\theta_{j}^{\prime}$ and the following distortion theorem of Teichmüller-Wittich-Belinskii. If $\phi$ is a homeomorphism of the plane, $\phi(\infty)=\infty$, whose dilatation

$$
p(z)=\left|\frac{\partial \phi / \partial \bar{z}}{\partial \phi / \partial z}\right|
$$

tends to zero fast enough, so that

$$
\iint_{|z|>1} p(z) \frac{d x d y}{|z|^{2}}<\infty
$$

then $\phi$ is conformal at infinity in the sense that there exists finite limit $\lim _{z \rightarrow \infty} \phi(z) / z \neq 0$.

Using this scheme, more and more general Riemann surfaces were treated (most of the results obtained before Gol'dberg's works are contained in Wittich's book [93]). Ullrich [82] introduced Riemann surfaces with finitely many periodic ends. A periodic end is the Riemann surface associated to a function of the form $h=P \circ \exp$ restricted to the upper half-plane, where $P$ is a rational function. The other types of ends considered by Künzi and others used restrictions of elliptic functions to certain angular sectors (doubly periodic ends and 1/4-ends). Constructions using elliptic functions were substantially generalized by Gol'dberg in [G16] where the so-called $\pi$-ends were studied.

In [G5] (see also [G57, Chapter VII]), Gol'dberg introduced a new class of Riemann surfaces which permitted him to give a complete solution of the inverse problem of Nevanlinna theory for functions of finite order with finite set of deficiencies. The crucial step made in this work was introduction of ends which are no longer explicitly defined using some special functions.

To explain Gol'dberg's contribution let us first consider a periodic end given by $h=P \circ \exp$ restricted to the upper half-plane $H$. The function $h$ has period $2 \pi i$ so its restriction to any strip $S_{k}=\{z: 2 \pi k \leq$ $3 z \leq 2 \pi(k+1)\}$ defines the same Riemann surface, namely the Riemann surface, associated to the rational function $P$ in the plane with a slit along 
the positive ray. Thus the periodic end can be thought as the result of pasting together infinitely many copies of Riemann surfaces of a single rational function $P$.

Gol'dberg considers more generally a sequence of rational functions $P_{k}, k=1,2, \ldots$. If $\gamma$ is a simple smooth curve which does not pass through the critical values of any $P_{k}$ then $R_{k}$ is defined as the Riemann surface associated to $P_{k}$ with a slit along some simple curve $\Gamma_{k}$ whose projection is $\gamma_{k}$. Then $R_{k}$ is pasted to $R_{k+1}$ for each $k$ the usual way along the edges of the slits $\Gamma_{k}$. The resulting Riemann surface is called $R$. If all $P_{k}$ are the same we have a periodic end. If $P_{k}$ are different, an explicit conformal map of a plane region onto $R$ cannot be found. But if the sequence $P_{k}$ satisfies some regularity conditions, a quasiconformal map of a half-plane onto $R$ can be found, and the asymptotic behavior of this quasiconformal map can be derived from the properties of the sequence $P_{k}$. When these regularity conditions are satisfied the resulting Riemann surface $R$ is called an almost periodic end.

The value of these constructions is not limited to their role in solution of the inverse problem of Nevanlinna theory, (see, for example [11]) and they certainly deserve to be better known.

These two classes of Riemann surfaces (almost periodic ends and $\pi$ ends) for long time remained the most general classes of parabolic simply connected Riemann surfaces for which the asymptotic behavior of the associated meromorphic function was known in detail. In 1975, another class of ends, which may be called Lindelöf ends, was introduced by Drasin and Weitsman [16] who used heavily Gol'dberg's technique from [G57, Chapter VII]. A Lindelöf end is the Riemann surface associated to the restriction on a sector $|\arg z|<\epsilon$ of the function $L \circ p$, where $L$ is a fractional-linear transformation and $p$ is the Hadamard canonical product with zeros $z_{k}=k^{\lambda}>0$. By pasting together finitely many logarithmic and Lindelöf ends, Drasin and Weitsman constructed meromorphic functions of finite order which are believed to be extremal for the defect relation in the class of functions of a given order.

\subsection{Comb-like entire functions}

An especially simple and useful class of Riemann surfaces can be obtained in the following way. Take infinitely many copies of the plane, call them $C_{k},-\infty<k<\infty$ and make two slits on each copy: from $-\infty$ to 
$-b_{k}<0$ and from $a_{k}>0$ to $+\infty$ such that $b_{k}=b_{k+1}$ for all even $k$ and $a_{k}=a_{k+1}$ for all odd $k$. Then paste $k+1$-th sheet to the $k$-th sheet along their common slit such that each endpoint of a slit becomes a simple branch point. It was proved by $G$. MacLane [56] that the resulting Riemann surface is always of parabolic type, see also the recent paper by Vinberg [86]. In fact the associated function can be always written in the form $f(z)=\exp \theta(z)$ where $\theta$ is the conformal mapping of the upper half-plane onto a region obtained from the plane by deleting some rays of the form $l_{k}=\left\{t+\pi i k:-\infty<t \leq c_{k}\right\}$ where $-\infty \leq c_{k}<\infty$ for each integer $k$ (here, $c_{2 k}=\log a_{k}, c_{2 k+1}=\log \left|b_{k}\right|$ ). The normalization is $\Re \theta(i y) \rightarrow+\infty, y \rightarrow+\infty$. This defines $f$ in the upper half-plane up to the real linear transformation of the argument and then it can be extended to the whole plane by symmetry.

This gives the geometric characterization of the Laguerre-Pólya class of entire functions, that is, entire functions which can be locally uniformly approximated by real polynomials with real zeros. The classical LaguerrePólya theorem [54] asserts that $f$ belongs to this class iff

$$
f(z)=e^{-\gamma z^{2}+\beta z+\alpha} z^{m} \prod_{n}\left(1-\frac{z}{z_{n}}\right) e^{z / z_{n}}
$$

where all $z_{n}, \alpha$ and $\beta$ are real, $\gamma \leq 0, m$ is a non-negative integer, and $\sum_{n}\left|z_{n}\right|^{-2}<\infty$. One can show that the Laguerre-Polya class coincides with the class of entire functions of the form $f(z)=\exp \theta(z)$ where $\theta$ is a conformal map onto a comb domain described above. ${ }^{3}$

For this special class of entire functions, the general problem stated in the introduction of section 2 becomes: to establish connections between the growth properties of $f$ and the sequence of its critical values $\left\{c_{k}\right\}$ (i.e., the sequence of branch points of the associated Riemann surface $F$ ).

The geometric characterization of Blaschke products with zeros on the positive ray obtained in [G6] (see Section 2.1) implies ${ }^{4}$ the necessary and sufficient condition on $\left\{c_{k}\right\}$ for $\gamma=0$ in the representation of $f(z)$, or, what is the same, for $f(z)$ to be a function of at most minimal type, order 2.

\footnotetext{
${ }^{3}$ Another type of comb domains is related to a more restricted class of real entire functions with real \pm 1 -points [59]. For the sake of definiteness, we restrict our discussion to the classes of comb domains and comb-like entire functions introduced above.

'It was Gol'dberg who paid our attention to this fact, which was not mentioned explicitly in [G6].
} 
The problem we are discussing is equivalent to a problem about asymptotic behaviour of the conformal mapping $\theta(z)$ of the upper halfplane onto a comb-like domain. Thus one can show for example that $f$ has exponential type and is bounded on the real line if and only if $\left\{c_{k}\right\}$ is a bounded sequence and the corresponding conformal map $\theta$ has an angular derivative. Using their new effective condition of the existence of angular derivative, Gentosh, Gol'dberg and Moravetskaya [G145] found necessary and sufficient conditions on $\left\{c_{k}\right\}$ which ensure that $f(z)$ is an entire function of exponential type bounded on the real axis.

Because of importance of comb-like entire functions and, especially, of their geometrical parameterization by critical values in applications (Chebyshev-type extremal problems and spectral theory of periodic and almost-periodic Sturm-Lioville operators), it would be highly interesting to have further results in this direction; for example, to find explicit formulas for the order of $f(z)$ in terms of the sequence $\left\{c_{k}\right\}$.

In [G67], Gol'dberg applied comb-like functions to construct a surprising example of entire function $f$ of arbitrary prescribed positive order such that the counting function $n(r)$ of all simple solutions of $f(z)= \pm 1$ satisfies $n(r)=O\left(\log ^{\sigma} r\right)$, with any given $\sigma>2$. This example is unexpected because according to an old theorem of $\mathrm{H}$. Selberg, ${ }^{5}$ entire functions whose all but finitely many \pm 1 -points are multiple has order which is a multiple of $1 / 2$. This example shows that Selberg's theorem is very unstable: permitting just "a few" simple \pm 1 -points drastically changes the picture. Also in this example we have $\varepsilon(1)=\varepsilon(-1)=1 / 2$ and $\delta(\infty)=1$ so that equality holds in (N5). This is in sharp contrast with the result of Pfluger that for entire functions $\sum \delta(a, f)=2$ implies that the order is a positive integer or infinite:

In [G132] a conjecture of one of the authors of this paper whose purpose was to give a characterization of the class of comb-like entire functions of exponential type belonging to $L^{p}$ on the real axis in geometric terms was disproved.

\section{Entire Functions}

The fundamental problem in the theory of entire functions is the problem of the connection between the growth of entire function and the distribution

\footnotetext{
${ }^{5}$ Another proof of Selberg's theorem was obtained by Gol'dberg and Tairova in [G34] by pure geometric methods; the original proof used differential equations and estimates of logarithmic derivatives.
} 
of its zeros [54]. The basic characteristic of growth of entire functions of finite order is the Phragmén-Lindelöf indicator. For entire function $f(z)$ of order $\rho$ and mean type the indicator is defined as

$$
h(\varphi, f)=\limsup _{r \rightarrow \infty} r^{-\rho} \log \left|f\left(r e^{i \varphi}\right)\right|, \quad 0 \leq \varphi<2 \pi .
$$

To deal with functions of maximal or minimal type with respect to the order $\rho, 0<\rho<\infty$ one should use the Valiron proximate order; i.e. a $C^{\prime}$-function $\rho(r)$ such that, for $r \rightarrow \infty$,

(i) $\rho(r) \rightarrow \rho$;

(ii) $\rho^{\prime}(r) r \log r \rightarrow 0$.

An entire function $f(z)$ is said to have a proximate order $\rho(r)$ if

$$
0<\limsup _{r \rightarrow \infty} r^{-\rho(r)} \max _{0 \leq \varphi<2 \pi} \log \left|f\left(r e^{i \varphi}\right)\right|<\infty .
$$

Then, the Phragmén-Lindelöf indicator is defined as

$$
h(\varphi, f)=\limsup _{r \rightarrow \infty} r^{-\rho(r)} \log \left|f\left(r e^{i \varphi}\right)\right|, \quad 0 \leq \varphi<2 \pi .
$$

The systematic study of the connection of the indicator with the distribution of zeros started in the 30-ies. In the years 1936-39, Levin and Pfluger independently defined and studied the class of entire functions of completely regular growth. For this class, the above-mentioned link can be written as equalities which express the indicator $h(\varphi, f)$ through the angular density of zeros

$$
\Delta(\alpha, \beta, f)=\lim _{r \rightarrow \infty} r^{-\rho(r)} n(r, \alpha, \beta, f),
$$

where $n(r, \alpha, \beta, f)$ is a number of zeros of $f(z)$ in the sector $\{z:|z| \leq$ $r, \alpha<\arg z \leq \beta$, and the limit exists for all but at most countable set of values $\alpha$ and $\beta$. An account of the theory of entire functions of completely regular growth is given in the Levin monograph [54], see also [6], [7], and [74].

\subsection{Connection between the Phragmén-Lindelöf indicator of entire function and its zero distribution}

In 1962-65, Gol'dberg published a cycle of papers [G28, G32, G33, $\mathrm{G} 35, \mathrm{G} 36, \mathrm{G} 42, \mathrm{G} 45, \mathrm{G} 50$ ] in which he studies the connection between 
the indicator and zero distribution in the general case of entire functions of finite order. In the general case, this connection cannot be written in the form of equalities; one deals with inequalities which give upper and lower bounds for the indicator through characteristics of the distribution of zeros. The starting point of this cycle was a theorem proved by Levin in [54, Chapter V, Theorem 12]: if $f(z)$ is an even canonical product of exponential type with real zeros

$$
f(z)=\prod_{n}\left(1-\frac{z^{2}}{a_{n}^{2}}\right)
$$

whose upper density of zeros equals

$$
\bar{\Delta}(f)=\limsup _{r \rightarrow \infty} r^{-1} n(r, f),
$$

then

$$
h(\varphi, f) \leq I \bar{\Delta}(f)
$$

where

$$
I=\left\{\begin{array}{l}
\int_{0}^{1 / \sqrt{(2 \cos \varphi)}} \log \left|1-\frac{e^{2 i \varphi}}{t^{2}}\right| d t \text { when }|\varphi|<\pi / 4 \text { or }|\varphi-\pi|<\pi / 4 \\
\pi|\sin \varphi| \\
\text { when }|\varphi-\pi / 2| \leq \pi / 4
\end{array}\right.
$$

(the integral can be computed, see [54, p.255]). Levin's estimate is sharp: there is a canonical product (13) of exponential type and with real zeros such that the equality holds in (14) for all $\varphi \in[0,2 \pi]$.

Let $E(z, p)$ be the Weierstrass primary factor, and let $K(t, \varphi, p)$ be the kernel given by

$$
K(t, \varphi, p)=-t^{p+1} \frac{d}{d t} \log \left|E\left(\frac{e^{i \varphi}}{t}, p\right)\right|=\frac{\cos p \varphi-t \cos (p+1) \varphi}{1-t \cos \varphi+t^{2}}
$$

at the points $(t, \varphi)$ at which both inequalities

$$
\log \left|E\left(e^{i \varphi} / t, p\right)\right|>0 \text { and } \frac{d}{d t} \log \left|E\left(e^{i \varphi} / t, p\right)\right|<0
$$

are satisfied, and equal to zero otherwise. Then Levin's estimate (14) can be restated as follows:

$$
h(\varphi, f) \leq \bar{\Delta}(f) \int_{0}^{\infty} t^{\rho-\rho-1} K(t, \varphi, p) d t \quad \text { for } \rho=1 / 2 .
$$


Estimate (15) was extended to entire functions of order $\rho \in(0,1)$ by Gol'dberg's Master Degree student Andrashko [3] and to entire functions of an arbitrary non-integer order by Gol'dberg himself [G32]. We mention that the sharp lower bound for the Phragmén-Lindelöf indicator of entire function of finite order with zeros on a ray remains unknown [72]. Another unsolved question concerns entire functions of finite order with zeros on a ray is the problem of description of their PhragmenLindelöf indicators, [72]. As Levin has told one of the authors, a similar question was asked by Mark G. Krein in the 30-ies.

To obtain estimates similar to (15) in the general case, one should drop the assumption that all zeros of $f(z)$ are on one ray. Let $f(z)$ be an entire function of proximate order $\rho(r)$. The upper angular density of zeros of $f(z)$ is defined as

$$
\bar{\Delta}(X, f)=\limsup _{r \rightarrow \infty} \frac{n(r, X, f)}{r^{\rho(r)}}
$$

where $X$ is an arbitrary subset of $[0,2 \pi]$ and $n(r, X, f)$ is a number of zeros of $f(z)$ in the sector $\{z:|z|=r, \arg z \in X\}$. Then $\bar{\Delta}(X, f)$ is a semi-additive measure, and the problem of finding of an adequate general version of (15) leads to an integral with respect to this semiadditive measure. Gol'dberg introduces such a new kind of integral which is interesting on its own (for an application outside Function Theory see [G128]).

Here we will give an equivalent definition of the Gol'dberg integral which was obtained later by Levin, Matsaev and Ostrovskii. To make this definition more transparent, we will consider a particular case only. Denote by $U$ the class of all subsets of $[0,2 \pi)$. A nonnegative, everywhere finite, set-function $\mu(X), X \in U$ is called a semi-additive measure if it satisfies the conditions:

(i) $\mu(\emptyset)=0$;

(ii) $\mu(X) \leq \mu(Y)$ if $X \subset Y$;

(iii) $\mu(X \cup Y) \leq \mu(X)+\mu(Y), \quad \forall X, Y \in U$.

If a set-function is initially defined only on the class $D$ of sets which are finite unions of disjoint intervals from $[0,2 \pi)$, then we can always extended it to $U$ by setting $\mu(X)=\inf \{\mu(Y): X \subset Y, Y \in D\}$. Let $M=M(\mu)$ be the class of all nonnegative (finitely-) additive measures $v$ such that

$$
\nu(X) \leq \mu(X), \quad \forall X \in U .
$$


For a non-negative function $f$, the Gol'dberg integral is defined as

$$
(U) \int_{[0,2 \pi)} f(x) d \mu(x)=\max _{\nu \in M} \int_{[0,2 \pi)} f(x) d \nu(x),
$$

the maximum in the right-hand side of (16) is attained.

With the help of this integral (16) Gol'dberg proves that, for noninteger $\rho$,

$$
\begin{aligned}
(U) & \int_{[0,2 \pi)} \int_{0}^{\infty} t^{\rho-[\rho]-1} K(t, \varphi-\theta,[\rho]) d t d \mu(\theta) \\
& \leq \sup \{h(\varphi, f): f \in F[\mu, \rho(r)]\} \\
& \leq \int_{0}^{\infty}(U) \int_{[0,2 \pi)} t^{\rho-[\rho]-1} K(t, \varphi-\theta,[\rho]) d \mu(\theta) d t
\end{aligned}
$$

where $F[\rho(r), \mu]$ is the class of all entire functions of proximate order $\rho(r)$ such that

$$
\bar{\Delta}(X, f) \leq \mu(X) \quad \forall X \in U .
$$

The integrals in the right- and left-hand sides of (17) differ by the order of integration. However, as examples show, they do not coincide in the general case. If $\mu$ is a countably-additive measure, then they coincide by the Fubini theorem.

Gol'dberg shows that there is an entire function $f(z)$ such that the lower bound

$$
h(\varphi, f) \geq(U) \int_{[0,2 \pi)} \int_{0}^{\infty} t^{\rho-[\rho]-1} K(t, \varphi-\theta,[\rho]) d t d \mu(\theta)
$$

holds simultaneously for all $\varphi \in[0,2 \pi)$. Levin's estimate (15) follows from (17). Another corollary deduced from (17) for arbitrary entire functions of proximate order $\rho(r) \rightarrow \rho, \rho$ non-integer, is the estimate

$$
h(\varphi, f) \leq \bar{\Delta}(f) \rho \int_{0}^{\infty} t^{\rho-1} M(1 / t,[\rho]) d t, \quad M(t, p)=\max _{|z|=t} \log |E(z, p)|
$$

which is also sharp: there is an entire function $f(z)$ of a prescribed proximate order such that equality holds in (18) simultaneously for all $\varphi \in[0,2 \pi)$.

Gol'dberg also obtained estimates of similar kind for entire functions of integral order. Besides, he considered more general problem to estimate the indicator and the lower indicator through the upper and lower angular densities of zeros. 
Making use of Azarin's theory of limit sets of subharmonic functions, Fainberg [27] (see also [7]) found another approach to Gol'dberg's results. She obtained a sharp upper bound for $h(\varphi, f)$ using a double Gol'dberg integral with respect to some semi-additive measure defined on $[0,2 \pi) \times[0, \infty)$ (instead of the repeated integral in (17)).

\subsection{Phragmén-Lindelöf indicators of Hermitian-positive entire functions}

Entire functions $f(z)$ represented in the form

$$
f(z)=\int_{-\infty}^{\infty} e^{i z u} d P(u)
$$

where $P$ is a probability measure on the line and the integral converges absolutely in $C$, are of a considerable interest for probability theory and, in particular for its part dealing with decompositions of random variable into the sum of independent random variables, see [55]. These functions are called entire Hermitian-positive.

In the works [G103, G115], the problem of description of PhragménLindelöf indicators of entire Hermitian-positive functions was completely solved. First, observe that all non-constant entire Hermitian-positive functions have a growth at least of order 1 and mean type.

Hence, denote by $I[\rho], l \leq \rho<\infty$, the class of real functions $h(\varphi)$ satisfying the conditions:

(i) $h(\varphi)$ is $2 \pi$-periodic $\rho$-trigonometrically convex function;

(ii) the function $h(\varphi+\pi / 2)$ is even;

(iii) $\max \{h(\varphi): 0 \leq \varphi<2 \pi\}=\max \{h(\pi / 2), h(-\pi / 2)\}>0$;

(iv) $h(\varphi) \leq h(\pi / 2)(\sin \varphi)^{\rho}$, for $0 \leq \varphi \leq \pi ;$ and $h(\varphi) \leq h(-\pi / 2)|\sin \varphi|^{\rho}$, for $-\pi \leq \varphi \leq 0$.

\section{THEOREM 8.}

(i) If $f(z)$ is an entire Hermitian-positive function of proximate order $\rho(r) \rightarrow \rho \geq 1$, then $h(\varphi, f) \in I[\rho]$; in addition, if

$$
\lim _{r \rightarrow \infty}(\rho(r)-1) \log r=+\infty
$$

then $h( \pm \pi / 2) \geq 0$.

(ii) Given $\rho, 1 \leq \rho<\infty$, a proximate order $\rho(r) \rightarrow \rho$, and a function $h(\varphi) \in I[\rho]$, there is an entire Hermitian-positive function $f(z)$ of proximate order $\rho(r)$, such that $h(\varphi, f) \equiv h(\varphi)$. In addition, if $\rho=1$ and $(20)$ holds, then $h( \pm \pi / 2) \geq 0$. 
The first statement of the theorem is not difficult, however, the second one is deep and technical. ${ }^{6}$

It tums out that the class $I[\rho]$ is rather wide. Denote by $v(\rho)$ the maximal possible number of intervals of negativity (i.e. components of the set $\{\varphi: h(\varphi)<0\}$ ) of indicator $h$ belonging to $I[\rho]$. The exact formula for $\nu(\rho)$ proved in [G103] shows that $\nu(\rho) \sim 2 \rho$ as $\rho \rightarrow \infty$. It means that $\nu(\rho)$ is asymptotically equal to the maximal possible number of intervals of negativity of a $2 \pi$-periodic $\rho$-trigonometrically convex function.

The class $I[\rho]$ for $\rho>1$ contains indicators $h$ such that $h(0)=$ $h(\pi)<0$. Thus, for every proximate order $\rho(r) \rightarrow \rho>1$, there are entire functions $f(z)$ of the form (19) rapidly decreasing along the real axis, so that $|f(x)|=O\left(\exp \left(-c|x|^{\rho(x)}\right)\right),|x| \rightarrow \infty, c>0$.

Theorem 8 has an application to an open problem going back to Marcinkiewicz [58] who proved that an entire Hermitian-positive function of finite order $\rho>2$ cannot have 0 as a Borel exceptional value. Kamynin and Ostrovskii [42] showed that such a function cannot have 0 as a Nevanlinna deficient value with deficiency 1 , but can have it with a positive deficiency. The problem arose to determine the value of

$$
C(\rho):=\sup \delta(0, f), \quad 2<\rho<\infty,
$$

\footnotetext{
${ }^{6}$ The proof of Theorem 8 utilized a new fact of an independent interest related to the theory of entire functions of completely regular growth in the Levin-Pfluger sense.

It immediately follows from the definition that the class of entire functions of completely regular growth of a fixed proximate order $\rho(r)$ is closed with respect to multiplication. Nevertheless. it is easy to construct examples showing that this class is not closed with respect to addition. Let $f_{1}, f_{2}$ be two functions of completely regular growth of a proximate order $\rho(r)$. Which can be the size of the set
}

$$
E\left(f_{1}, f_{2}\right)=\left\{a \in C: f_{1}+a f_{2} \text { does not have completely regular growth }\right\} \text { ? }
$$

In [G103], it is proved that $E\left(f_{1}, f_{2}\right)$ is of logarithmic capaciry zero. This result was improved in [G 105] where it was shown that $E\left(f_{1}, f_{2}\right)$ is $H$-set in the sense of Hyllengren. Another result obtained in [G105] says: there are entire functions of completely regular growth having uncountable many Valiron deficient values. A little bit later, using the methods of Drasin's paper [12], Eremenko [22] characterized the set of Valiron deficient values of entire functions of completely regular growth.

A related question posed in [G103] was the starting point for Favorov who developed in [28] a theory of holomorphic curves in Banach spaces (see also his subsequent paper [29]). Making use of this theory, Favorov proved in [28] the following: Let $f_{n}$ be a sequence of entire functions of completely regular growth of the same proximate order $\rho(r)$ such that, for all $\varphi, h(\varphi)=\sup _{n} h_{f_{n}}(\varphi)<\infty$. Then there is a sequence $b_{n}>0$ such that the entire function $f(z)=\sum_{n} b_{n} f_{n}(\xi)$ is of completely regular growth with respect to the proximate order $\rho(r)$ and has indicator $h(\varphi)$. We do not know a simple proof of this result. 
where the supremum is taken over all entire Hermitian-positive functions $f$ of order $\rho$. In [42] it has been proved that, for large $\rho$,

$$
A_{1} \rho^{-\rho}<C(\rho)<1-\frac{A_{2}}{\rho \log \rho},
$$

where $A_{1}, A_{2}$ are positive constants. As it is shown in [G103], Theorem 8 yields that $C(\rho)$ tends to $l$ as $\rho \rightarrow \infty$, moreover,

$$
1-C(\rho)=O(1 / \sqrt{\rho}), \quad \rho \rightarrow \infty .
$$

The connection between the Phragmen-Lindelöf indicator of a function of the form (19) and behaviour of the measure $P$ still remains mistly. However, the case of order $\rho=1$ is trivial since functions of the class $I[1]$ must have the form $h(\varphi)=c_{1} \sin \varphi$, as $0 \leq \varphi \leq \pi$, and $h(\varphi)=c_{2} \sin \varphi$, as $0 \geq \varphi \geq \pi$, where constants $c_{1}, c_{2}$ are related to the measure $P$ in a simple way.

A related study appears in [G127], where the authors considered Phragmén-Lindelöf indicators of entire functions of finite order represented by the Dirichlet series

$f(z)=\sum_{k} a\left(\lambda_{k}\right) \exp \left(z \lambda_{k}\right), \quad 0 \leq \lambda_{1}<\lambda_{2}<\cdots<\lambda_{k} \uparrow \infty, a\left(\lambda_{k}\right) \in C$.

\section{References}

[1] Ahlfors, L. (1932). Über eine in der neueren Wertverteilungstheorie betrachtete Klasse transzendenter Funktionen. Acta math., 58, Collected papers, 1, 112-143.

[2] Anderson, J. M. and Clunie, J. (1966). Slowly growing meromorphic functions. Comment. Math. Helv., 40, 267-280.

[3] Andrashko, M. I. (1960). Extremal indicator of entire functions of order less than one with positive zeros. Dopovidi AN URSR, (7), 869-872, (Russian).

[4] Arakelyan, N. (1966). Entire functions of finite order with an infinite number of defective values. Doklady AN SSSR, 170, 999-1002. Engl. transl.: Soviet Math. Dokl., 7.

[5] Arakelyan, N. (1970). Entire and analytic functions of bounded growth with an infinite number of defective values. $L v$. AN Armyan. SSR, 5, 486-506.

[6] Azarin, V.S. On asymptotic behaviour of subharmonic functions of finite order. Mat. Sb., 108, (1979), 147-164, (Russian); Engl. transl.: Math. USSR Sb., 36, (1980), $135-154$.

[7] Azarin, V. S. (1997). Limit sets of entire and subharmonic functions. In: Entire and meromorphic functions. Complex Analysis. One Variable, 1. Encyclopaedia of Math. Sci., Springer-Verlag. Berlin et al.

[8] Baemstein, A. II. (1978). How the *-function solves extremal problems. In: Proc. Intern. Congress of Math., Helsinki, II, 639-644.

[9] Baernstein, A. II. (1988). Ahlfors and conformal invariants, Ann. Acad. Sci. Fenn. Ser. A. I. Math., 13, pp. 289-312. 
[10] Barry, P. D. (1962). The minimum modulus of small emtire functions. Proc. London Math. Soc., 12, 445-495.

[11] Clunie, J., Eremenko, A. and Rossi, J. (1993). On equilibrium points of logarithmic and Newtonian potentiais, J. London Math. Soc., 47(2), 309-320.

[12] Drasin, D. (1974). Value distribution of entire functions in the regions of small growth. Ark. mat., 12, 139-150.

[13] Drasin, D. (1977). The inverse problem of the Nevanlinna theory. Acta Math., 138, . 83-151.

[14] Drasin, D. (1987). Proof of a conjecture of F. Nevanlinna concerning functions which have deficiency sum two. Acta math., 158, 1-94.

[15] Drasin, D. (1994). Meromorphic functions: progress and problems. In: Proc. Intern. Congress of Math., Zürich, II, pp. 812-835.

[16] Drasin, D. and Weitsman, A. (1975). Meromorphic functions with large sums of deficiencies, Adv. math., 15, 93-126.

[17] Dufresnoy, J. (1939). Sur les fonctions méromorphes dans un angle. C. R. Acad. Sci., 208, 718-720.

[18] Dufresnoy, J. (1944). Théorie nouvelle des familles complexes normales. Ann. sci. École Norm. Sup., 61, 1-44.

[19] Dugue, D. (1947). Le defaut au sens de $M$. Nevanlinna dépend de l'origine choisie. Comptes Rendus Acud. Sci., 225, 555-557.

[20] Edrei, A. and Fuchs, W. H. J. (1959). On the growth of meromorphic functions with several deficient values. Trans. Amer. Math. Soc., 93, 292-328.

[21] Eremenko, A. (1984). Meromorphic solutions of first-order differential equations. Functional Analysis and its Applications, 18, 246-248.

[22] Eremenko, A. On Valiron deficient values of entire functions of completely regular growth. Function Theory, Functional Analysis and their Applications (Kharkov), 44, (1985), 48-52, (Russian); Engl. transl.: J. of Soviet Math., 48, (1989).

[23] Eremenko, A. (1987). On the set of deficient values of an entire function of finite order. Ukr. Mat. Zh., 39, 295-299, (Russian); Engl. transl.: Ukrainian Math. Journal, 39, 225-228.

[24] Eremenko, A. (1986). The inverse problem of the theory of meromorphic functions. Sibirsk. Math. Zh., 27, 87-102, (Russian); Engl. transl.: Siber. Math. J., 39.

[25] Eremenko, A. and Sodin, M. Value distribution of meromorphic functions and meromorphic curves from the point of view of potential theory. Algebra and Analiz, 3. (1991), 131-164, (Russian); Engl. uransl.: St. Petersburg Math. J., 3, (1992), 109-136.

[26] Essén, M. (1975). The $\cos \pi \lambda$-theorem. Lecture Notes in Math., Springer Verlag, Berlin, 467.

[27] Fainberg, E. D. (1983). An integral with respect to a non-additive measure and estimates of the indicator of an entire function. Sibirsk. Mat. Zh., 24, 175-186, (Russian); Engl. transl.: Siber. Math. J., 24.

[28] Favorov, S. Yu. (1990). Growth and distribution of zeros of holomorphic mappings of a finite-dimensional space into a Banach space. Sibirsk. Mat. Zh., 31, 161-171, (Russian); Engl. transl.: Siber. Math. J., 31.

[29] Favorov, S. Yu. (1994). On growth of holomorphic mappings from finite-dimensional spaces into Banach spaces. Math. Physics, Analysis and Geometry, (Kharkov), 1, 240-25l, (Russian).

[30] Fenton, P. C. (1982). The minimum modulus of certain small entire functions. Trans. Amer. Math. Soc., 271, 183-195.

[31] Fuchs, W. H. J. and Hayman, W. K. (1962). An entire function with assigned deficiencies. Studies in Mathematical tralysis and related topics. Stanford University Press, pp. 117-125.

[32] Gleizer, E. (1986). Growth of entire function with zeros on a system of rays. Ukr. Mat. Zh., 38, 297-302, (Russian); Engl. transl.: Ukrain. Math. J., 38, 256-261. 
[33] Green, M. (1972). Holomorphic maps into complex projective space omitting hyperplanes. Trans. Amer. Math. Soc., 169, 89-103.

[34] Grishin, A. Ph. (1994). Nevanlinna theory for functions meromorphic in the upper half-plane. Manuscript.

[35] Hayman, W. K. (1953). An integral function with a defective value that is neither asymptotic nor invariant unider change of origin. J. London Math. Soc., 28, 369-376.

[36] Hayman, W. K. (1964). Meromorphic functions. Oxford and Clarendon Press.

[37] Hayman, W. K. (1978). On Iversen's theorem for meromorphic functions with few poles. Acta Math., 141, 115-145.

[38] Hayman, W. K. (1989). Subharmonic functions, Academic Press, New York, II.

[39] Hellerstein, S. and Shea, D. (1978). Minimal deficiencies for entire functions with radially distributed zeros and poles. Proc. London. Math. Soc., 37, 35-55.

[40] Heins, M. (1957). Asymptotic spots of entire and meromorphic functions. Ann Math., 66, 430-439.

[41] Hinkkanen, A. (1992). A sharp form of Nevanlinna's second fundamental theorem. Invent. Math. 108, 549-574.

[42] Kamynin, I. and Ostrovskii, I. (1975). On zeros of entire ridge functions. Function Theory, Functional Analysis and their Applications (Kharkov), 24, 42-50, (Russian)

[43] Keldysh, M. V. (1954). On series in rational functions. Dokl. Acad. Nauk SSSR, 94 377-380, (Russian).

[44] Bo Kjellberg (1948). On certain integral and harmonic functions. A study in minimum modulus. Thesis, Uppsala University, Uppsala.

[45] Kolokol'nikov, A. S. (1974). The logarithmic derivative lemma of a meromorphic function. Math. Notes, 15, 425-429.

[46] Laine, I. (1993). Nevanlinna theory and complex differential equations. de Gruyter Studies in Mathematics, de Gruyter, Berlin, 15.

[47] Lang, S. (1988). The error term in Nevanlinna theory. Duke Math. J., 56, 193-218.

[48] Le-Van-Thiem (1949). Uber das Umkehrproblem der Wertverteilungslehre. Comment. Math. Heiv., 23, 26-49.

[49] Le-Van-Thiem (1950). Sur une problème d'inversion dans la théorie des fonctions méromorphes. Ann. Sci. École Norm. Sup., 67, 51-98.

[50] Lehto, $O$. (1953). On an extension of the concept of deficiency in the theory of meromorphic functions. Math. Scand., 1, 207-212.

[51] Lehto, 0 . (1954). On the distribution of values of meromorphic functions of bounded characteristics. Acta Math., 91, 87-112.

[52] Lehto, $O$. (1954). Value distribution and boundary behavior of a function of bounded characteristic and the Riemann surface of its inverse function. Ann. Acad. Sci. Fenn. $177(\mathrm{~A} 1), 1-46$

[53] Lehto, O. and Virtanen, K. (1965). Quasikonforme Abbildungen, Springer, Berlin.

[54] Levin, B. Ja. (1980). Distribution of zeros of entire functions. Amer. Math. Soc., Providence, RI.

[55] Linnik, Yu. V. and Ostrovskii, I. V. (1977). Decomposition of random variables and vectors. Amer. Math. Soc., Providence.

[56] MacLane, G. Riemann surfaces and asymptotic values associated with real entire functions. Rice Institute Pamphlet, November, 1952.

[57] Malmquist, J. (1920). Sur les fonctions à un nombre fini de branches satisfaisant à une équation différentielle du premier ordre. Acta math, 42, 59-79.

[58] Marcinkiewicz, J. (1938). Sur une propriété de la loi de Gauss. Math. Zeitschr., 44, 612-618.

[59] Marchenko, V. A. and Ostrovskii, I. V. (1975). A characterization of the spectrum of Hill's operator. Mat. Sb., 97, 540-606, (Russian); Engl. transl.: Math. USSR Sb., 26, 493-554

[60] Miles, J. (1979). On entire functions of infinite order with radially distributed zeros. Pacif. J. Math., 81, 131-157. 
[61] Miles, J. (1983). Some examples of dependence of the Nevanlinna deficiency upon the choice of the origin. Proc. London Math. Soc., 47, 145-176.

[62] Miles, J. (1986). On the growth of meromorphic functions with radially distributed zeros and poles. Pacif. J. Math., 122, 131-157.

[63] Miles, J. (1992). A sharp form of the lemma on the logarithmic derivative. J. London Math. Soc., 45, 243-254.

[64] Narasimhan, R. (1971). Un analogue holomorphe du théorème de Lindemann. Ann. Inst. Fourier, 21, 271-278.

[65] Nevanlinna, R. (1925). Über die Eigenschaften meromorpher Funktionen in einem Winkelraum, Acta Soc. Sci. Fenn., 50(12), 1-45.

[66] Nevanlinna, R. (1929). Le theorème de Picard-Borel et la théorie des fonctions méromorphes. Paris, Gauthier-Villars.

[67] Nevanlinna, R. (1932). Uber Riemannsche Fläche mit endlich vielen Windungspunkten. Acta Math., 58, 295-373.

[68] Nevanlinna, R. (1936). Eindeutige analytische Funktionen. Berlin, Springer Verlag.

[69] Vu Ngoan and Ostrovskii, I. V. (1965). The logarithmic derivative of a meromorphic function. Dokl. Acad. Nauk Arm. SSR, 41, 272-277, (Russian).

[70] Osgood, Ch. (1985). Sometimes effective Thue-Siegel-Roth-Nevanlinna bounds, or better. J. Number Th., 21, 347-399.

[71] Ostrovskii, I. V. (1961). Connection between the growth of meromorphic functions and the distribution of its values. Izv. AN SSSR, Ser. Matem., 25, 277-328, (Russian).

[72] Ostrovskii, I. V. and Sodin, M. (1994). Problems 16.4 and 16.5. In: Linear and Complex Analysis Problem Book 3. Part II. V. P. Havin and N. K. Nikolski (Eds). Lecture Notes in Math., Berlin et al, Springer Verlag, 1574.

[73] Pólya, G. Zur Untersuchung der Grössenordnung ganzer funktionen die einer Differentialgleichung genügen, Acta math., 42, (1920), 309-316; Collected Papers, 1, p. 123.

[74] Ronkin, L. I. (1992). Functions of completely regular growth. Kluwer, Dordrecht.

[75] Min Ru and Stoll, W. (1991). The second main theorem for moving targets. J. Geom. Analysis, 1, 99-138.

[76] Schwartz, M.-H. (1941). Exemple d'une fonction méromorphe ayant des valeurs déficientes non asymptotiques. Comptes Rendus Acad. Sci., 212, 382-384.

[77] Steinmetz, N. (1983). On the order and lower order of entire functions with radially distributed zeros. Proc. Amer. Math. Soc., 87, 449-452.

[78] Teichmüller, O. (1939). Vermutungen und Sätze über die Werverteilung gebrochener Funktionen endlicher Ordnung. Deutsche Math., 4, 163-190.

[79] Teichmüller, O. Einfache Beispiele zur Wertverteilungslehre. Deutsche Math., 7. (1944), 360-368. Ges. Abh., S. 728.

[80] Toda, N. Sur les combinaisons exceptionnelles de fonctions holomorphes; applications aux fonctions algébroides. Tohoku Math. J., 22(2), (1970), 290-319; Sur le nombre de combinaisons exceptionnelles; applications aux fonctions algébroides. ibid, 480-491: Sur une généralisation du théorème de Picard-Borel-Rémundos. ibid, 23, (1971), 105-115.

[81] Toda, N. (1974/5). Sur quelques combinaisons linéares exceptionnelles au sense de Nevanlinna, III. Kodai Math. Sem. Rep., 26, 294-303.

[82] Ullnich, E. (1936). Zum Umkehrproblem der Wertverteilungslehre. Nachr. Ges. Wiss. Göttingen. Math.-Phys. Kl., 1, 135-150.

[83] Valiron, G. (1954). Fonctions Analytiques, Presses Universitaires de France, Paris.

[84] Valiron, G. (1947). Valeurs exceptionelles et valeurs déficientes des fonctions méromorphes. Comptes Rendus Acad. Sci., 225, 556-558.

[85] Valiron, G. (1950). Sur la valeurs déficientes des fonctions méromorphes. Comptes Rendus Acad. Sci., 230, 40-42.

[86] Vinberg. E. (1989). Real entire functions with prescribed critical values. Problems in Group Theory and in Homological Algebra, Yaroslavl'. Gos. Univ.. Yaroslavl", pp. 127-138, (Russian). 
[87] Vojta, P. (1987). Diophantine approximations and value distribution theory, Lect. Notes Math., Springer, Berlin er al, 1239.

[88] Volkovyskii, L. I. (1950). Researches in the type problem of a simply connected Riemann surface. Proceedings of Steklov Inst., 34, (Russian).

[89] Weitsman, A. (1972). A theorem on Nevanlinna deficiencies. Acta math., 128, 41 -52.

[90] Wiman, A. (1905). Sur une extension d'une théorème de M. Hadamard. Arkiv för Mat. Astr. Fys., 2(14), 1-5.

[91] Wittich, H. (1948). Zum Beweis eines Satzes über quasikonforme Abbildungen. Math. Zetschr., 51, 278-288.

[92] Wittich, H. (1948). Uber eine Klasse meromorpher Funktionen. Arch. Math., 1, $160-166$.

[93] Wittich, H. (1955). Neuere Untersuchungen über eindeutige analytische Funktionen, Springer, Berlin.

\section{Publications of A. A. Gol'dberg?}

[GI] On a problem in the theory of distribution of values of meromorphic functions. DAN URSR (1954), no.1, 3-5. (Ukrainian) MR 16, 1095.

[G2] (with P. P. Belinskii) Application of a theorem on conformal mappings to questions of invariance of defects of meromorphic functions. UMZ 6 (1954), no.3, 263-269. (Russian) MR 16, 1095.

[G3] On the influence of clustering of algebraic branch points of a Riemann surface on the order of growth of a meromorphic mapping function. DAN SSSR 98 (1954), no.5, 709-711. (Russian) MR 17, 144.

[G4] On defects of meromorphic functions. DAN SSSR 98 (1954), no.6, 893-895. (Russian) MR 17, 144 .

[G5] On the inverse problem of the theory of the distribution of the values of meromorphic functions. UMZ 6 (1954), no.4, 385-397. (Russian) MR 16, 1095.

[G6] Some asymptotic properties of meromorphic functions. Uchenyje Zapiski L'vovsk. Gos. Univ.. Ser. Meh.-Mat., 38 (1956), no.7, 54-74, (Russian).

[G7] On single-valued integrals of first-order differential equations. UMZ 8 (1956), no.3, 254-261. (Russian) MR 19 \#32.

[G8] An example of an entire function of finite order with a non-asymptotic defective value. Nauchn. Zapiski Uzhgorodsk. Gos. Univ. 18 (1957), 191-194. (Russian) Engl. transl.: AMS Transl. (2) 18 (1961), 45-47. MR 20 \#11.

[G9] An inequality for logarithmically convex functions. DAN URSR, (1957), no. 3 , 227-230. (Ukrainian) MR 19 \#950.

[G10] An estimate of the defects of meromorphic function of order less than one. DAN SSSR 114 (1957), no.2, 245-248. (Russian) MR 19 \#737.

[GII] Some bounds for meromorphic functions of order less than one. DSUGU (1957), no.1, 12, (Russian).

[G12] Some asymptotic properties of entire functions of several variables. DSUGU (1958), no.2, 19. (Russian).

${ }^{7}$ The following abbreviations of names of Russian and Ukrainian journals are used below: DAN SSSR = Doklady Akademii Nauk SSSR; DAN URSR = Dopovidi Akademii Nauk Ukrains'koi RSR; UMZ = Ukrainskii Matematicheskii Zhournal; TFFA = Teoria Funktsii, Funktsional'nyj Analiz i ih Prilozhenia (Kharkov); UMN = Uspekhi Matematicheskih Nauk: MSb = Matematicheskii Sbornik; IZVUZ = Izvestia Vysshikh Uchebnykh Zavedenij. Matematika: SMZ = Sibirskii Matematicheskii Zhournal; DSUGU = Doklady i Soobshenia Uzhgorodskogo Gosudarstvennogo Universiteta, seria fizika-matematiknhimia. 
[GI3] On entire solutions of partial differential equations. IZVUZ (1958), no.3, 62-66. (Russian) MR 24 \#A1495.

[GI4] (with I. F. Bitlyan) The Wiman-Valiron theorems for entire functions of several complex variables. Vestnik Leningrad. Univ., Ser. Matematika, Mekh., Astron. (1959), no. 13, 27-41. (Russian) MR 22 \#5735.

[GI5] A remark on Blaschke products for the half-plane. UMZ 11 (1959), no.2, 210-213. (Russian) MR 22 \#2712.

[G16] On a class of Riemann surfaces. MSb 49 (1959), no.4, 447-458. (Russian) MR 22 $\# 5730$.

[GI7] On the set of deficient values of meromorphic functions of finite order, UMZ 11 (1959), no.4, 438-443. (Russian) MR 24 \#A235.

[Gi8] Elementary remarks to formulas defining order and type of entire functions of several variables. Akad. Nauk Armyansk. SSR Dokl. 29 (1959), 145-151. (Russian) MR 23 \#A1053.

[G19] Meromorphic functions with separated zeros and poles. IZVUZ (1960), no.4, 67-72. (Russian) MR 24 \#A1400.

[G20] A theorem of Liouville type. UMN 15 (1960), no.5, 155-158. (Russian) MR 23 $\#$ \#3248.

[G21] On meromorphic functions with separated zeros and poles. DSUGU (1960), no.3, 72, (Russian).

[G22] Contemporary research on the Nevanlinna theory of distribution of values of meromorphic functions of finite order. In: Investigations on contemporary problems of Complex Analysis (A. I. Markushevich, ed.). Fizmatgiz. Moscow. 1960. pp.407-417. (Russian); French transl.: Fonctions d'une variable complexe. Problemes contemporains. Gauthier-Villars. Paris. 1962. MR 23 \#A332.

[G23] Some problems of the value distribution theory. Translator's addendum to the Russian edition of the book by Hans Wittich Neuere Untersuchungen über eindeutige analytische Funktionen. Fizmatgiz. Moscow. 1960. pp.263-300. (Russian) MR 23 \#A333.

[G24] (with M. V. Shvalagin) Summation of certain series by means of the theory of residues. UMZ 13 (1961), no.2, 217-220. (Russian) MR 26 \#521.

[G25] Distribution of values of meromorphic functions with separated zeros and poles. DAN SSSR 137 (1961), no.5, 1030-1033. (Russian); Engl. transl.: Soviet Math. Dokl. 2 (196I), 389-392. MR 229601.

[G26] On formulas for calculation of order and type of entire functions of several variables. DSUGU (1961), no.4, 101-103, (Russian).

[G27] A remark to the Tumura-Clunie theorem. DSUGU (1961), no.4, 109-110, (Russian).

[G28] Integral with respect to a semi-additive measure. DSUGU (196l), no.4, 104-105, (Russian).

[G29] Contribution to a theorem of M. G. Krein. DSUGU (1961), no.4, 106-108, (Russian).

[G30] (with I. V. Ostrovskii) Some theorems on the growth of meromorphic functions. Zapiski mat. otdelenija fiz-mat. fakul'teta Khar'kovsk. Gos. Univ. i Khar'kovsk. mat. obshchestva 27 (1961), 3-37, (Russian).

[G3I] (with I. V. Ostrovskii) New investigations on the growth and distribution of values of entire and meromorphic functions of genus zero. UMN 16 (1961), no.4, 5I-62. (Russian) MR 24 \#A2034.

[G32] Extremal indicator for an entire function with positive zeros. SMZ 3 (1962), no.2, 170-177. (Russian); Engl. transl.: Siber. Math. J. 3 (1962). MR 26 \#1458.

[G33] Extremal indicator for entire functions of entire order. DSUGU (1962), no.5, 85-87, (Russian).

[G34] (with V. G. Tairova) On analytic functions with two finite completely ramified values. DSUGU (1962), no.5, 87-88, (Russian).

[G35] Integral with respect to a semi-additive measure and its application to the theory of entire functions, I. MSb 58 (1962), no.3, 289-334. (Russian); Engl. transl.: AMS Transl. 88 (1970), 105-162. 
[G36] Integral with respect to a semi-additive measure and its application to the theory of entire functions, II. MSb 61 (1963), no.3, 334-349. (Russian); Engl. transl.: AMS Transl. 88 (1970), 163-180. MR $29 \# 1335$.

[G37] (with V. G. Tairova) On entire functions with two finite completely ramified values. Zapiski mat. otdelenia fz-mat. fakul'teta Khar'kovsk. Gos. Univ. i Khar'kovsk. mat. obshchestva 29 (1963), 67-78, (Russian).

[G38] Three examples of entire functions. DAN URSR (1963), no.4, 443-446, (Ukrainian).

[G39] On the growth of an entire function along a ray. DAN SSSR 152 (1963), no.5, 1049-1050. (Russian); Engl. transl.: Soviet Math. Dokl. 4 (1963). 1491-1493. MR 27 \#4933.

[G40] On the lower order of an entire function with a finite defective value. SMZ 5 (1964), no.1, 54-76. (Russian); Engl. transl.: Siber. Math. J. 5 (1964). MR 28 \#4114.

[G41]. A conformal sewing problem for half-strips. UMZ 16 (1964), no.5, 586-592. (Russian) MR 30 \#3967.

[G42] Integral with respect to a semi-additive measure and its application to the theory of entire functions, III. MSb 65 (1964), no.3, 414-453. (Russian); Engl. transl.: AMS Transl. 88 (1970), 181-232. MR 32 \#212.

[G43] (with B. Ya. Levin) Entire functions which are bounded on the real axis. DAN SSSR 157 (1964), no.1, 19-21. (Russian); Engl. transl.: Soviet Math. Dokl. 5 (1964). 859-861. MR 29 \#2394.

[G44] On the possible values of the lower order of an entire function with a finite deficient value. DAN SSSR 159 (1964), no.5, 968-970. (Russian); Engl. transl.: Soviet Math. Dokl. 5 (1964), 1631-1633. MR 30 \#247.

[G45] An integral with respect to a semi-additive measure and its application to the theory of entire functions, IV. MSb 66 (1965), no.3, 411-457. (Russian); Engl. Transl.: AMS Transl. 88 (1970), 233-289. MR 34 \#7798.

[G46] The growth of an entire function along a ray. TFFA 1 (1965), 164-175. (Russian) MR $33 \# 7539$

[G47] Distribution of values of the Weierstrass sigma-function. IZVUZ (1966), no.l, 43-46. (Russian) MR 34 \#1526.

[G48] On deficient non-asymptotic values of meromorphic functions. In: Abstracts of short sci. reports of Moscow Internat. Math. Congress 1966, Sec.4. Moscow. 1966. p.47, (Russian).

[G49] Non-asymptotic defect values of meromorphic functions. DAN SSSR 171, (1966), no.2, 254-257. (Russian); Engl. transl.: Soviet Math. Dokl. 7 (1966), 1444-1447. MR 34 \#2887.

[G50] Indicators of entire functions and integral with respect to a non-additive measure. In: Contemporary problems in the theory of analytic functions. Nauka. Moscow. 1966. pp. 88-93. (Russian) MR $34 \# 6089$

[G51] (with T. V. Strochik) Conformal representation of symmetric half-strips and angular domains. Litovsk. Mat. Sb. 6 (1966), no.2, 227-239. (Russian) MR 35 \#593.

[G52] Non-asymptotic defect values of entire functions of infinite order. Mat. Zametki 1 (1967), no.1, 81-86. (Russian) MR 34 \#7802.

[G53] (with I. V. Ostrovskii) Application of W. K. Hayman's theorem to a problem of the theory of decomposition of probability laws. UMZ 19 (1967), no.3, 104-106. (Russian); Engl. transl.: Selected Transl. in Math. Stat. and Probab., vol.9. AMS. Providence. 1970. pp.147-151. MR 35 \#7366.

[G54] On a functional equation. TFFA 5 (1967), 34-36. (Russian) MR 37 \#1600.

[G55] The distribution of values of an entire function with respect to arguments. Acta math. Acad. scient. hungaricae, 19 (1968), no.1-2, 191-199. (Russian) MR 37 \#3393.

[G56] (with F. A. Baraket) Defecticve non-asymptotic values of meromorphic functions of order one. SMZ 10 (1969), no.3, 483-487. (Russian); Engl. transl.: Siber. Math. J. 10 (1969), 345-348. MR $40 \# 4458$.

[G57] (with I. V. Ostrovskii) The distribution of values of meromorphic functions. Nauka. Moscow. 1970. 592 pp. (Russian) MR $43 \# 6439$. 
[G58] (with B. Ya. Levin and I. V. Ostrovskii) General theory of entire and meromorphic functions. In: History of the native mathematics, vol.4, part 1. Naukova Dumka. Kiev. 1970. pp.9-48, (Russian).

[G59] (with G. S. Prokopovich) The primality of certain entire functions. UMZ 22 (1970), no.6, 813-817. (Russian); Engl. transl.: Ukrain. Math. J. 22 (1970), 701-705. MR 45 \#2169.

[G60] (with S. B. Tushkanov) Exceptional combinations of entire functions. TFFA, 13 (1971), 67-74. (Russian) MR $45 \# 7056$.

[G61] (with M. N. Sheremeta) The fix-points of entire functions. Visnik L'viv. Derzh. Univ., Ser. Meh.-Mat. (1971), no.6, 5-8. (Ukrainian) MR 47 \#3349.

[G62] The defects of entire functions of completely regular growth. TFFA 14 (1971), $88-101$. (Russian) MR 45 \#7057.

[G63] On entire functions without finite Valiron defect values. TFFA 15 (1972), 244-254. (Russian) MR 46 \#3792.

[G64] The representation of a meromorphic functions as a quotient of entire functions. IZVUZ (1972), no.10, 13-17. (Russian) MR 47 \#855.

[G65] Meromorphic functions. In: Results of Science and Technique. Mathematical Analysis, vol.10, VINITI, Moscow, 1973, pp.5-97. (Russian); Engl. transl.: J. Soviet Math. 4 (1975), pp.157-216. MR $51 \# 8421$.

[G66] A certain theorem of Landau type. TFFA 17 (1973), 200-206. (Russian) MR 50 \#4951.

[G67] The branched values of entire functions. SMZ 14 (1973), no.4, 862-866. (Russian); Engl. transl.: Siber. Math. J. 14 (1973), 599-602. MR 49 \#584.

[G68] On a problem of Yu. V. Linnik. DAN SSSR 211 (1973), no.1, 31-34. (Russian); Engl. transl. Soviet Math. Dokl. 14 (1973), 950-953. MR 48 \#7337.

[G69] (with I. V. Ostrovskii) The derivatives and the primitives of entire functions of completely regular growth. TFFA 18 (1973), 70-81. (Russian) MR 50 \#4945.

[G70] (with S. B. Tushkanov) Addendum to the paper "On exceptional combinations of entire functions". TFFA 18 (1973), 185-189. (Russian) MR 49 \#3131.

[G71] (with V. S. Boichuk) On the three lines theorem. Mat. Zametki 15 (1974), no.l, 45-53. (Russian); Engl. transl: Math. Notes 15 (1974), 26-30. MR 49 \#9204.

[G72] A certain problem of P. Turán. Izv. Akad. Nauk UzSSR, Ser. Fiz-Mat nauk (1974), no.3, 19-23. (Russian) MR 50 \#2495.

[G73] Boolean algebras with derivatives. UMZ 26 (1974), no.4, 444-449. (Russian); Engl. transl.: Ukrain. Math. J. 26 (1974), 365-369. MR 51 \#2917.

[G74] (with I. V. Ostrovskii) The growth of entire ridge functions with real zeros. In: Mathematical Physics and Functional Analysis, no.5. FTINT AN USSR. Kharkov. 1974. pp.3-10. (Russian); Engl. transl.: Selected Transl. in Math. Stat. and Probab., vol.15. AMS. Providence. 1981. pp.147-155. MR 53 \#13561.

[G75] Nevanlinna's lemma on the logarithmic derivative of a meromorphic function. Mat. Zametki 17 (1975), no.4, 525-530. (Russian); Engl. transl.: Math. Notes 17 (1975), 310-312. MR 53 \#816.

[G76] (with A. Z. Mokhon'ko) The rate of growth of the solutions of algebraic differential equations in angular domains. Diff. Uravn. 11 (1975), no.9, 1568-1574. (Russian); Engl. transl.: Diff. Eq. 11 (1975), 1169-1173. MR 57 \#16768.

[G77] (with T. V. Strochik) On the conformal representation of some types of half-strips. Litovsk. Mat. Sb. 16 (1976), no.2, 43-49. (Russian) MR $54 \# 10617$.

[G78] (with V. A. Grinshtein) The logarithmic derivative of a meromorphic function. Mat. Zametki 19 (1976), no.4, 525-530. (Russian); Engl. transl.: Math. Notes 19 (1976), 320-323. MR $53 \# 13561$.

[G79] (with M. B. Balk) An improved version of the big Picard theorem for polyanalytic functions. UMZ 28 (1976), no.4, 435-442. (Russian); Engl. transl.: Ukrain. Math. J. 28 (1976), 337-342. MR 54 \#10617.

[G80] (with M. B. Balk) A strengthening of the big Picard theorem for bianalytic functions. In: Mathematical Analysis and Theory of Functions, no.7, Moscow, 1976, pp.82-88. (Russian) MR 58 \#28542. 
[G81] (with V. D. Mokhon'ko) Generalized Nevanlinna characteristics. lzv. Akad. Nauk Armyan. SSR, Ser. Mat. 11 (1976), no.2, 132-154. (Russian) MR 54 \#2968.

[G82] (with M. B. Balk) Functions that are polyanalytic in a disc. IZVUZ (1977), no.5, 3-14. (Russian) MR 58 \#22594.

[G83] On the question of the connection between the defect and the deviation of a meromorphic function. TFFA 29 (1978), 31-35. (Russian) MR 81b: 30058.

[G84] Counting functions of sequences of a-points for entire functions. SMZ 19 (1978), no. 1, 28-36. (Russian); Engl. transl.: Siber. Math. J. 19 (1978), 19-25. MR 57 \#6428.

[G85] (with N. E. Korenkov) The asymptotic behavior of the logarithmic derivative of an entire function of completely regular growth. UMZ 30 (1978), no.1, 25-32. (Russian); Engl. transl.: Ukrain. Math. J. 30 (1978), 17-22. MR 58 \#6237.

[G86] The growth of meromorphic solutions of second order differential equations. Diff. Uravn. 14 (1978), no.5, 824-829. (Russian); Engl. transl.: Diff. Eq. 14 (1978), 584-588. MR $58 \# 11412$.

[G87] (with N. E. Korenkov) Asymptotic behavior of the logarithmic derivative of an entire function of order zero. UMZ 30 (1978), no.3, 291-298. (Russian); Engl. transl.: Ukrain. Math. J. 30 (1978). MR 58 \#17086.

[G88] (with L. S. Kochinyan and S. Ts. Sarkisyan) Some theorems of Picard type. lzv. Akad. Nauk Armyansk. SSR, ser. Mat. 13 (1978), no.1, 23-35. (Russian); Engl. transl.: Sov. J. Contemp. Math. Anal. 13 (1978). MR 58 \#11413.

[G89] Sets on which the modulus of an entire function has a lower bound. SMZ 20 (1979), no.3, 512-518. (Russian); Engl. transl.: Siber. Math. J. 20 (1979), 360-364. MR 81c: 30059.

[G90] (with A. N. Fridman) Regular growth of meromorphic functions with positive zeros and negative poles. Vestnik L'vovsk. Gos. Univ., Mat. analiz i jego prilozh. (1979), no.14, 13-18. (Ukrainian) MR 84f: 30040.

[G91] The minimum of modulus of a meromorphic function of slow growth. Mat. Zametki 25 (1979), no.6, 835-844. (Russian); Engl. uransi.: Math. Notes 25 (1979), 432-437. MR 81c: 30058 .

[G92] Defective values of the derivative of a meromorphic function with the maximal sum of defects. TFFA 32 (1979), 28-30. (Russian) MR 80j: 30038.

[G93] (with A. E. Eremenko) Asymptotic curves of entire functions of finite order. MSb 109 (1979), no.4 555-581. (Russian); Engl. transl.: Math. USSR, Sb. 37 (1980), 509-533. MR 81c: 30057.

[G94] (with M. N. Sheremeta) The ridge property of the superposition of an entire function and an exponential polynomial. Akad. Nauk Armyan. SSR Dokl. 69 (1979), no.3. 129-134, (Russian).

[G95] The sum of defects of a meromorphic function and its derivative. TFFA 33 (1980), 37-40. (Russian) MR 81h: 30030.

[G96] (with N. E. Korenkov) The defect at zero of the logarithmic derivative of an entire function. TFFA 34 (1980), 41-46. (Russian) MR 81d: 30048.

[G97] (with N. E. Korenkov) Asymptotic behaviour of the logarithmic derivative of an entire function of completely regular growth. SMZ 21 (1980), no.3, 63-79. (Russian); Engl. transl.: Siber. Math. J. 21 (1981), 363-367. MR 82c: 30041 .

[G98] On the growth of functions meromorphic in a disc with restrictions on its logarithmic derivative. UMZ 32 (1980), no.4, 456-462. (Russian); Engl. transl.: Ukrain. Math. J. 32 (1980), 311-316. MR 82c: 30045.

[G99] A remark on bounded analytic functions. Visnyk L'vivs'k Derzh., Pytannya mat. analizu ta jogo zastosuv. (1980), no.18, 68-70, (Ukrainian).

$[\mathrm{Gl00}]$ (with I. V. Ostrovskii) The growth of entire Hermitian-positive functions of finite order. DAN URSR, ser.A (1981), no.4, 6-9. (Ukrainian) MR 82j: 30033.

[G101] Defects of an entire function of completely regular growth and of its derivative. IZVUZ (1981), no.10, 10-14. (Russian) MR 83g: 30027.

[G102] (with A. E. Eremenko and I. V. Ostrovskii) On the sum of entire functions of completely regular growth. DAN URSR, ser.A (1982), no.2, 8-11. (Ukrainian) MR 83f: 30025 . 
[G103] (with I. V. Ostrovskij) Indicators of entire Hermitian-positive functions of finite order. SMZ 23 (1982), no.6, 55-73. (Russian); Engl. transl.: Siber. Math. J. 23 (1983), 804-820. MR 84d: 30042.

[G104] (with N. V. Zabolotskii) On a-points of functions meromorphic in a disc. SMZ 24 (1983), no.3, 34-46. (Russian); Engl. transl.: Siber. Math. J., 24 (1984), 342-352. MR 85a: 30048.

[G105] (with A. E. Eremenko and 1. V. Ostrovskii) On a sum of entire functions of completely regular growth. Izv. Akad. Nauk Armyan. SSR, Ser. Mat. 18 (1983), no.l, 3-14. (Russian); Engl. transl.: Sov. J. Contemp. Math. Anal. 18 (1983), no.1, 1-12. MR 85a: 30042 .

[G106] (with N. V. Zabolotskii) Index of concentration of a subharmonic function of zero order. Mat. Zametki 34 (1983), no.2, 227-236. (Russian); Engl. transl.: Math. Notes 34 (1984), 596-601. MR 85a: 31006.

[G107] The growth of an entire function representable by a gap power series. TFFA 41 (1984), 37-39. (Russian) MR 85k: 30045.

[G108] (with L. A. Karaseva) Creative elements in a lecture. Vestnik Vysshej shkoly (1984), no.2, 24-25, (Russian).

[G109] Analytic functions mapping a disc onto itself. IZVUZ (1984), no.6, 24-25. (Russian); Engl. transl.: Soviet Math. (Izv. VUZ) (1984). MR 86a: 30012.

[G110] (with A. E. Eremenko and M. L. Sodin) Defects and deviations of meromorphic functions of finite order. DAN URSR, ser.A (1984), no.10, 3-5. (Ukrainian) MR 86d 30046.

[G111] (with I. V. Ostrovskii) Paley's phenomenon for entire characteristic functions and entire functions representabie by Dirichlet series. TFFA, 44 (1985), 18-23. (Russian); Engl. transl.: J. Sov. Math. 48 (1989), 255-259. MR 86m: 30029.

[Gi12] (with N. N. Strochik) Asymptotic behavior of meromorphic functions of completely regular growth and of their logarithmic derivatives. SMZ 26 (1985), no.6, 29-38. (Russian); Engl. transl.: Siber. Math. J. 26 (1985), 802-809. Corrigendum, SMZ 33 (1992), no.4, 219. (Russian); Engl. transl.: Siber. Math. J. 33 (1992), 742.) MR 87c: 30043.

[G113] (with E. V. Gleizer) On an extremal problem connected with harmonic measure. Analysis mathematica 11 (1985), 23-28. MR 86i: 49013.

[G114] (with A. E. Eremenko) Generalized sets of uniqueness for entire functions. Akad. Nauk Armyan. SSR Dokl. 81 (1985), no.4, 159-161. (Russian) MR 87j: 30050.

[G115] (with I. V. Ostrovskii) Indicators of entire absolutely monotone functions of finite order. SMZ, 27 (1986), no.6, 33-49. (Russian) Engl. transl.: Siber. Math. J. 27 (1986), 811-825. MR 89a: 30024.

[G116] (with A. E. Eremenko and M. L. Sodin) Exceptional values in the sense of R. Nevanlinna and in the sense of V. P. Petrenko, I. TFFA 47 (1987), 41 -52. (Russian) Engl. transl.: J. Sov. Math. 48 (1990), no.6, 648-655. MR 88m: 30075a.

[G117] (with A. E. Eremenko and M. L. Sodin) Exceptional values in the sense of R. Nevanlinna and in the sense of V. P. Petrenko, II. TFFA 48 (1987), 58-70. (Russian) Engl. transl.: J. Sov. Math. 49 (1990), no.2, 891-899. MR 88m: 30075b.

[G118] (with O. P. Sokolovskaya) Some relations for meromorphic functions of order or lower order less than one. I IZVUZ (1987), no.6, 26-31. (Russian); English transl.: Soviet Math. (Izv. VUZ) 31 (1987). MR 88h: 30042.

[G119] (with O. P. Sokolovskaya) On the radial growth of a subharmonic function whose mass distributed along the negative half-axis. Manuscript no.2293-Uk87, UkrNIINTI 03.08.87, Kiev, 1987, 16 pp, (Russian).

[G120] (with O. P. Sokolovskaya) Growth on a ray of a subharmonic function whose mass distributed on the negative half-axis. TFFA 50 (1988), 31-38. (Russian) MR 90c: 31002.

[G121] Integral representation of monotone slowly varying functions. IZVUZ (1988), no.4, 21-27. (Russian); English transl.: Soviet Math. (Izv. VUZ) 32 (1988). MR 89h: 26012. 
[G122] Distribution of values of meromorphic functions with poles attracted to a system of rays. UMZ 41 (1989), no.6, 738-743. (Russian); English transl.: Ukrain. Math. J. 41 (1989), 634-638. MR 90e: 30029.

[G123] (with Sh. Yu. Lomsadze) Asymptotic correlations of the type of Pomeranchuk's theorem for non-microcausal amplitudes. Short commun. in Physics, FIAN SSSR (1989), no.5, 16-18, (Russian).

[GI24] (with I. V. Ostrovskii) Indicators of entire functions of finite order represented by Dirichlet series. DAN URSR, ser.A (1990), no.1, 13-15. (Ukrainian) MR 91g: 30001.

[G125] (with O. P. Sokolovsknya) Some relations for meromorphic functions of order or lower order less than one. II IZVUZ (1990), no.5, 25-32. (Russian); English transl.: Soviet Math. (Izv. VUZ) 34 (1990). MR 91k: 3007 I.

[G126] (with I. M. Tkach) A remark on Pólya peaks. IZVUZ (1990), no.6, 25-26. (Russian); English transl.: Soviet Math. (Izv. VUZ) 34 (1990), 28-30.

[G127] (with 1. V. Ostrovskii) Indicators of entire functions of finite order represented by Dirichlet series. Algebra i Analiz 2 (1990), no.3, 144-170. (Russian); Engl. transl. Leningr.Math. J. 2 (1991). 589-612. Corrigendum: Algebra i Analiz 2 (1990), no.6, 195-196. (Russian) MR 91h: 30046.

[G128] An integral with respect to a fuzzy measure. Iry. Akad. Nauk Armyan. SSR, Ser. Mat. 25 (1990), no.4, 361-373. (Russian) English transl.: Soviet J. Contemp. Math. Anal. 25 (1991), 49-62. MR 92g: 28042.

[G129] (with B. Ya. Levin and I. V. Ostrovskii) Entire and meromorphic functions. In: Complex Analysis. One Variable. Results of Science and Technique. Contemporary Problems of Mathematics. Fundamental Directions, vol.85. VINITI. Moscow. 1991. pp.5-186. (Russian); English transl.: Complex Analysis. One variable 1, Encyclopaedia of Math. Sci., Springer-Verlag, Berlin (to appear) MR 93a: 30031.

[G130] (with M. N. Sheremeta) Analytic Functions. Minvuz URSR. Kiev. 1991. 116 pp. (Ukrainian).

[GI31] (with L. A. Ajzenberg et al.) Lev Isaakovich Ronkin (to 60th birthday). UMN, 46 (1991), no.5, 181-183. (Russian); English transl.: Russian Math. Surveys 46 (1991), 207-209. MR 93e: 01016.

[G132] On a condition for an entire function of exponential type to belong to the class $L^{P}$. Matemarychni studii (Papers of the Lviv Mathematical Society) 1 (1991), 55-60. (Ukrainian) MR 93j: 30025

[G133] (with M. M. Zarichnyj and B. I. Ptashnik) On the history of Ukrainian mathematical culture in Galicia. In: Essays on the history of natural science and technique, no.40. "Naukova Dumka". Kiev. 1991. pp.8-13. (Russian) MR 93f: 01015.

[G134] Application of C. Carathéodory's theorem to a problem of the theory of entire functions. In: Constantin Carathéodory: An International Tribute; vol.I. World Scientific Publ. Co. Singapore 1991. pp.374-380. MR 93f: 30029.

[G135] (with V. A. Pyana) Uniqueness theorems for algebraic functions. In: Adv. in Soviet Math. Il. AMS. Providence RI 1992. pp.199-204. MR 94f: 14020.

[G136] (with V. S. Azarin) Review: W. K. Hayman and P. B. Kennedy. Subharmonic functions, vol. 1, 1976; W. K. Hayman. Subharmonic functions, vol.2. 1989. Algebra $i$ Analiz 4 (1992), no.1, 194-201. (Russian); Eng!. transl.: St. Peresburg Math. J. 4 (1993).

[G137] (with M. L. Sodin and N. N. Strochik) Meromorphic functions of completely reguler growth and their logarithmic derivatives. SMZ 33 (1992), no.1, 44-52. (Russian); Engl. transl.: Siber. Marh. J. 33 (1992), 34-40. MR 93e: 30065.

[G138] (with V. A. Pyana) Some uniqueness theorems for rational, algebraic and algebroid functions taking into account only simple a-points. Dokl. Akad. Nauk Ukrainy (1992), no.12, 13-15. (Russian) MR 93k: 30044.

[G139] (with I. E. Sheikhet) Comparison of the characteristics of growth of functions in the sense of Pólya and Kondratyuk. In: Dynamical Systems and Complex Analysis "Naukova Dumka”, Kiev, 1992, pp.54-63. (Russian) MR 94h: 30038.

[GI40] Contemporary situation in the theory of functions of a complex variable. In: Philosophical problems in teaching of mathematical disciplines. L'viv State University, L'viv. 1993. pp.32-37, (Ukrainian). 
[G141] (with M. N. Sheremeta) Analytic continuation to the whole plane of functions analytic in the unit disc. TFFA 58 (1993), 21-30. (Russian) MR 95g: 30004.

[G142] (with M. Gorbachuk et al.) Ivan Georgievich Sokolov (in memoriam). UMZ 45 (1993), no.8, 1184. (Ukrainian); Engl. transl.: Ukr. Math. J. 45 (1993), 1325.

[G143] (with V. S. Azarin et al.) Boris Yakovlevich Levin (obituary). UMN 49 (1994), no.1, 201 -202. (Russian); Engl. transl.: Russian Math. Surveys 49 (1994).

[G144] Existence and some properties of meromorphic functions for which the equality sign in the deficiency relation of Yang Lo is attained. Matematychni Studii (Papers of the Lviv Mathematical Society) 3 (1994), 53-60, (Ukrainian).

[G145] (with O. E. Gentosh and O. N. Moravetskaya) Geometric criteria of the existence of strip and angular derivatives for some classes of conformal mappings. Mathematical Physics, Analysis, Geometry (Kharkov) 1 (1994), no.1, 102-115, (Russian).

[G146] B. Ya. Levin is the creator of the theory of entire functions of completely regular growth. Mathematical Physics, Analysis, Geometry (Kharkov) 1 (1994), no.2, 186-192, (Russian).

[G147] (with V. A. Pyana) Some uniqueness theorems for rational, algebraic and algebroid functions. UMZ 46 (1994), no.3, 212-226. (Russian); English transl.: Ukrain. Mat. J., 46 (1994), 219-235. MR 96a: 30036.

[G148] (with M. N. Sheremeta) On the existence of an entire transcendental function of bounded $l$-index. Mat. Zametki 57 (1995), no.1, 125-129. (Russian); English transl.: Math. Notes 57 (1995), 88-90. MR 96e: 30066.

[G149] Uniqueness theorems for algebraic functions taking into account the number of algebraic elements. UMZ 47 (1995), no.11, 1498-1505. (Russian); English transl.: Ukrain. Mat. J., 47 (1995).

[G150] (with V. S. Azarin et al.) lossif Vladimirovich Ostrovskii (to 60th birthday). UMN 50 (1995), no.2, 232-235. (Russian); English tranls.: Russian Math. Surveys 50 (1995).

[G151] A bound for the modulus of the logarithmic derivative of the Mittag-Leffler function and its application. Matematychni Studii (Papers of the Lviv Mathematical Sociery) 5 (1996), 21-30, (Ukrainian).

\section{Gol'dberg's Ph. D. Students}

[1] Tairova, V. G. (1964). On some problems of the theory of Riemann surfaces.

[2] Geche, F. I. (1965). Some theorems on the growth of entire functions and vectorvalued functions of several complex variables and their applications.

[3] Strochik, T. V. (1969). Conformal representation of half-strips.

[4] Kondratyuk, A. A. (1969). Bounds for indicators of entire functions.

[5] Sheremeta, M. N. (1969). Connection between the asymptotic behavior of analytic functions and coefficients of their power series expansions.

[6] Prokopovich, G. S. (1974). Distribution of values and fix-points of superpositions of meromorphic functions.

[7] Gimyk. M. A. (1977). Asymptotic properties of some classes of functions analytic or subharmonic in a disc.

[8] Mokhon'ko, A. Z. (1978). Some applications of the theory of analytic functions to differential equations and planar curves.

[9] Mokhon'ko, V. D. (1978). Generalizations and applications of the lemma on the logarithmic derivative.

[10] Eremenko, A. E. (1979). Asymptotic properties of meromorphic and subharmonic functions.

[11] Korenkov, N. E. (1979). Asymptotic properties of the logarithmic derivatives of entire functions.

[12] Fridman, A. N. (1981), Lower bounds of analytic and subharmonic functions.

[13] Zabolotskii, N. V. (1982). Asymptotic properties of meromorphic and $\delta$-subharmonic functions of the slow growth. 
[14] Gleizer, E. V. (1988). Asymptotic behavior of meromorphic and $\delta$-subharmonic functions with a special distribution of values and masses.

[15] Sokolovskaya, O. P. (1989). Asymptotic relations for $\delta$-subharmonic functions of order or lower order less than one.

[16] Strochik, N. N. (1991). The growth of several quantities characterizing asymptotic properties of meromorphic functions.

[17] Pyana, V. A. (1993). Uniqueness theorems for algebraic and algebroid functions.

\section{Sci. Theses defended by students of A. A. Gol'dberg}

[1] Sheremeta, M. N. (1987). Asymptotic properties of entire functions given by power and Dirichlet series.

[2] Eremenko, A. E. (1987). Asymptotic properties and value distribution of meromorphic functions.

[3] Kondratyuk. A. A. (1988). Method of Fourier and Fourier-Laplace series for meromorphic and subharmonic functions of completely regular growth.

[4] Mokhon'ko, A. Z. (1994). New relations between the Nevanlinna characteristics and their applications. 